\title{
Neural Characterization of the Speed-Accuracy Tradeoff in a Perceptual Decision-Making Task
}

\author{
Hermine Wenzlaff, ${ }^{1,2}$ Markus Bauer, ${ }^{3}$ Burkhard Maess, ${ }^{4}$ and Hauke R. Heekeren ${ }^{1,2}$ \\ ${ }^{1}$ Max Planck Institute for Human Development, 14195 Berlin, Germany, ${ }^{2}$ Cluster Languages of Emotion, Freie Universität Berlin, 14195 Berlin, Germany, \\ ${ }^{3}$ Wellcome Trust Centre for Neuroimaging, University College London, London WC1N 3BG, United Kingdom, and ${ }^{4}$ Max Planck Institute for Human \\ Cognitive and Brain Sciences, 04303 Leipzig, Germany
}

Decisions often necessitate a tradeoff between speed and accuracy (SAT), that is, fast decisions are more error prone while careful decisions take longer. Sequential sampling models assume that evidence for different response alternatives is accumulated over time and suggest that SAT modulates the decision system by setting a lower threshold (boundary) on required accumulated evidence to commit a response under time pressure. We investigated how such a speed accuracy tradeoff is implemented neurally under different levels of sensory evidence. Using magnetoencephalography (MEG) and a face-house categorization task, we show that the later decision-and motor-related systems rather than the early sensory system are modulated by SAT. Source analysis revealed that the bilateral supplementary motor areas (SMAs) and the medial precuneus were more activated under the speed instruction and correlated negatively (right SMA) with the boundary parameter, whereas the left dorsolateral prefrontal cortex (DLPFC) was more activated under the accuracy instruction and showed a positive correlation with the boundary. The findings are interpreted in the sense that SMA activity dynamically facilitates fast responses during stimulus processing, potentially by disinhibiting thalamo-striatal loops, whereas DLPFC reflects accumulated evidence before response execution.

\section{Introduction}

Every day when making decisions, e.g., when driving the car or playing ball, we trade off speed against accuracy (SAT), that is, fast decisions are more error prone while careful ones take longer (Bogacz et al., 2010). But how does SAT affect our decisions and how is it modulated under different levels of sensory evidence?

Perceptual decision making is defined as a process where a sensory stimulus is presented upon which a subject has to choose from several alternative interpretations and commit the choice via an overt motor response (Shadlen and Newsome, 2001; Gold and Shadlen, 2007). Thereby, distinct but interacting systems can be differentiated: the "sensory system" representing the sensory evidence; the "decision system" integrating the sensory evidence and forming a decision variable; and the "motor system" representing the decision variable and executing a motor response (Heekeren et al., 2008).

In the context of sequential sampling models, decisions are viewed as a continuous accumulation of sensory information from a starting point until a boundary is reached (Ratcliff, 1978). Reaching the boundary concludes the decision process and initiates a response. Whereas the accumulation rate is determined by the quality of the sensory representation, the boundary can be set according to emphasis on speed or accuracy of the decision (Bogacz et al., 2006; Ratcliff and McKoon, 2008).

Received July 31, 2010; revised 0ct. 14, 2010; accepted Nov. 8, 2010.

This research was supported by the Max Planck Society and by a grant from the German Research Foundation (Deutsche Forschungsgemeinschaft; HE 3347/2-1). We thank Yvonne Wolff for assistance with data acquisition.

Correspondence should be addressed to Prof. Dr. Hauke Heekeren, Freie Universität Berlin, Cluster Languages of Emotion, Habelschwerdter Allee 45, 14195 Berlin, Germany. E-mail: hauke.heekeren@fu-berlin.de.

DOI:10.1523/JNEUROSCI.4000-10.2011

Copyright $\odot 2011$ the authors $\quad 0270-6474 / 11 / 311254-13 \$ 15.00 / 0$
Several studies have shown how manipulations of sensory evidence alter decision processing in the brain (Kim and Shadlen, 1999; Heekeren et al., 2004, 2006; Philiastides et al., 2006) involving a network of sensory, parietal, and frontal areas.

Three recent functional magnetic resonance imaging (fMRI) studies have identified brain regions involved in SAT (Forstmann et al., 2008; Ivanoff et al., 2008; van Veen et al., 2008). They suggest that SAT affects the decision and motor rather than the sensory system by modulating activity in association cortices including the dorsolateral prefrontal cortex (DLPFC), posterior lateral prefrontal cortex, and parietal areas, as well as in premotor areas including pre-supplementary motor area (pre-SMA), SMA, and striatum.

Several electroencephalography (EEG) studies have addressed the temporal dynamics of neural processes underlying SAT (Osman et al., 2000; Sangals et al., 2002; Rinkenauer et al., 2004). However, they primarily focused on motor preparation and motor execution processes by analyzing the lateralized readiness potential. Hence, no inferences about the behavior of more distributed networks underlying decision processing, e.g., about the involvement of sensory systems, could be deduced from these studies.

In the present study, using magnetoencephalography (MEG), we investigated at which temporal stages and in which neuroanatomical structures SAT affects decision making, and whether that depends on sensory evidence. Subjects discriminated pictures of faces or houses presented under speed or accuracy instructions and at different levels of sensory evidence. We hypothesized that speed versus accuracy instructions might alter the level of activity in structures that have previously been associated with decision processing, such as the DLPFC (Kim and Shadlen, 1999; Heekeren et al., 2004). Further, we hypothesized that neurophysiological SAT effects should correlate with parameters from sequential sampling models. 
A
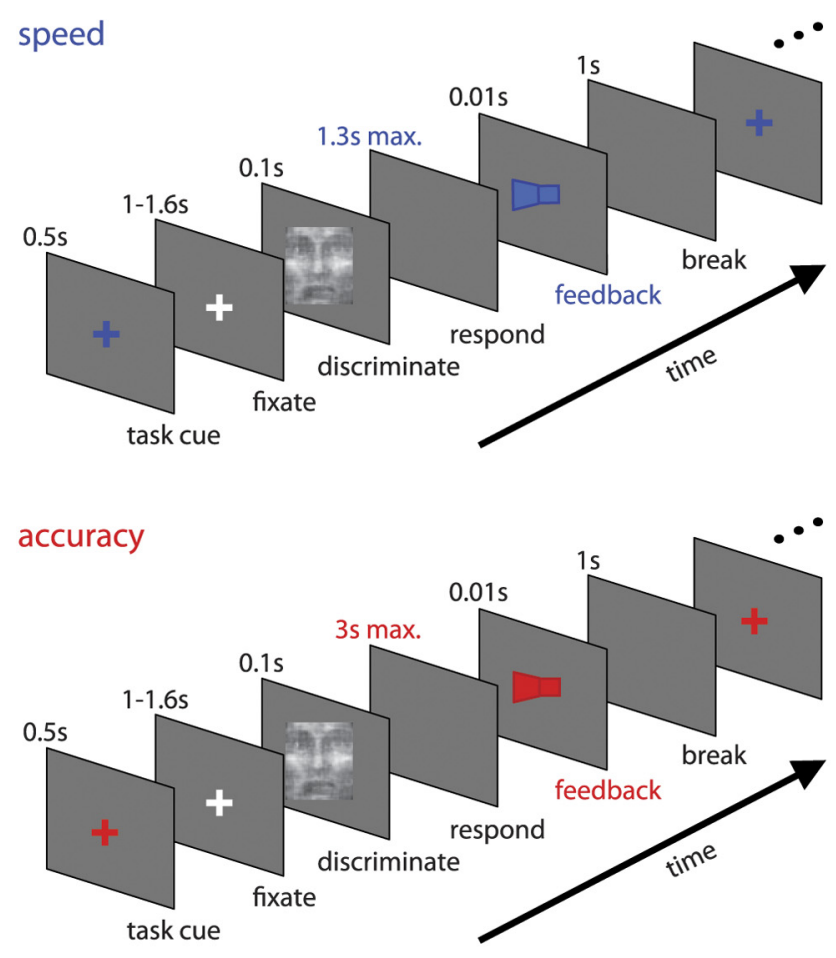

B

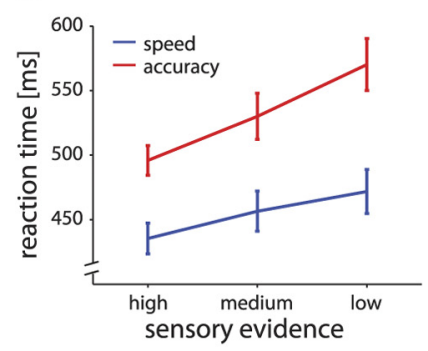

C
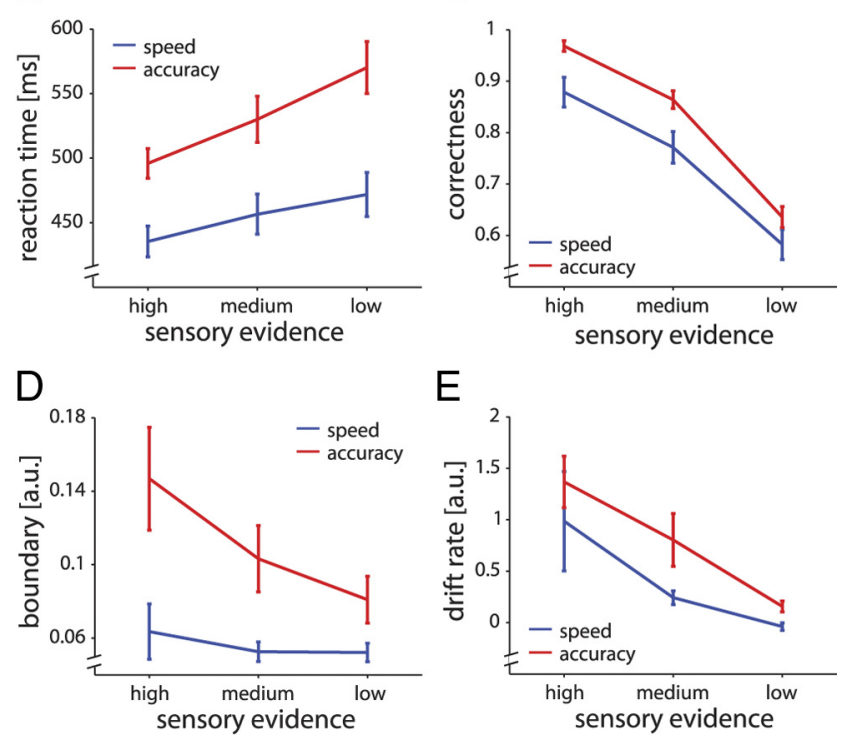

E

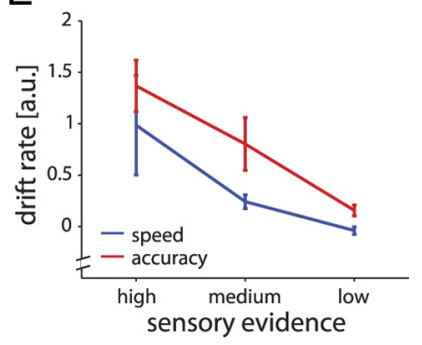

Figure 1. Experimental design, behavioral and modeling results. $\boldsymbol{A}$, Images of faces or houses at three different levels of sensory evidence $(\mathrm{COH})$ were presented in random order. At each trial, initially one of two differently colored task cues (cross) indicated whether subjects should answer as fast or as accurately as possible (SAT). Feedback, a low-frequency tone, was given when subjects responded slower than their expected mean $\mathrm{RT}$ corresponding to the particular $\mathrm{COH}$ level presented (speed instruction) or incorrectly (accuracy instruction) (see Materials and Methods for further details). $\boldsymbol{B}-\boldsymbol{E}$, Under the speed compared with the accuracy instruction, subjects showed significantly faster RTs (B) $\left(F_{(1,9)}=21.42, p=0.0012\right)$ and lower correctness $(C)\left(F_{(1,9)}=9.89, p=0.012\right)$. Furthermore, the boundary of the diffusion model was significantly lower under speed than accuracy conditions $(\boldsymbol{D})\left(F_{(1,9)}=10.35, p=0.011\right)$ but was not significantly different for either the drift rate $\left(F_{(1,9)}=1.55, p=0.23\right)$ or the nondecision time $(\boldsymbol{E})\left(F_{(1,9)}=\right.$ $2.57, p=0.14)$. With decreasing $\mathrm{COH}$, subjects showed slower RTs $(B)\left(F_{(2,18)}=30.52\right.$, $p<0.001)$, higher error rates $(C)\left(F_{(2,18)}=104.43, p<0.001\right)$, lower boundaries $(\boldsymbol{D})$ $\left(F_{(2,18)}=4.89, p=0.020\right)$ (for explanation see Discussion), and, as expected, lower drift rates $\left(F_{(2,18)}=11.27, p<0.001\right)$ as well as no significant differences in nondecision time $(\boldsymbol{E})\left(F_{(2,18)}=\right.$

\section{Materials and Methods}

\section{Subjects}

Twelve subjects (six females; age range 20-30 years) participated in the study, after having provided written informed consent in accordance with the guidelines and approval of the Max Planck Institute for Human Development, Berlin, Germany. All had normal or corrected to normal vision and no reported history of neurological problems.

\section{Stimuli}

We used the same set of 36 grayscale images of faces and houses used by Heekeren et al. (2004). Fast Fourier transformation was applied to separate amplitude and phase matrices of each image. Amplitudes of all images were averaged resulting in an identical frequency spectrum for all images. The phase matrix of each image was scrambled by adding a random offset of $0-100 \%$ (in $1 \%$ steps) to the original phase matrix. Inverse fast Fourier transformation of the average amplitude and individual phase matrices resulted in 36 face and 36 house images in different levels of sensory evidence [coherence $(\mathrm{COH})]$ that were used for the training and experiment.

For feedback about their performance (cf. below), subjects heard highor low-frequency tones via an MEG-compatible headphone system (Tip300 , Viasys Healthcare) at $50 \mathrm{~dB}$.

Visual and auditory stimulation was controlled by an IBM-compatible computer and Presentation software (Neurobehavioral Systems Inc.). To present the images $\left(3.44 \times 4.01^{\circ}\right.$ visual angle), a projector with digital light processing (Panasonic Corporation) was used with a $1024 \times 768$ resolution and a $60 \mathrm{~Hz}$ refresh rate. Pictures were shown in the center of a back projection screen (Elekta Neuromag) with a 0.6 gray filter and an illumination of 10.30 .

Task

Subjects performed a two-alternative forced-choice task. Pictures of faces and houses were presented and subjects indicated their subjective report via an MEG-compatible response box by pressing the left button with their left thumb when they perceived a face and the right button with their right thumb when they perceived a house.

Subjects were tested on three successive days. In the training session (day 1), face and house stimuli were presented at six different $\mathrm{COH}$ levels $(41,44,47,50,54,58 \%)$. They were instructed to respond as fast and as accurately as possible within $3000 \mathrm{~ms}$. They received auditory feedback after each trial: a pleasant high-frequency tone for a correct and an unpleasant low-frequency tone for an incorrect choice.

To define three $\mathrm{COH}$ levels for the experiment according to the individual performance levels, we computed a maximum-likelihood fit of a cumulative Weibull function: $p=1.0-0.5^{\star} e^{-\left(x / \alpha_{1}\right)^{\beta}}$, where $p$ is the proportion of correct choices, $x$ is the spatial phase coherence level of the stimuli, and $\alpha_{1}$ and $\beta_{1}$ are free parameters of the function fitted to the data. We used the FitWeibTAFC.m and FitWeibAlphTAFC.m functions of the Psychophysics Toolbox (Brainard, 1997) to estimate $\alpha_{1}$ and $\beta_{1}$. With these values we computed the psychometric function (WeibAlphTAFCFitFun.m) to estimate the spatial phase coherence levels of the face and house stimuli corresponding to the individual performance levels of 95,82 , and $70 \%$ correctness.

To estimate the three average reaction times (RTs) for the above chosen spatial phase coherence levels (matching individual performance levels), we fitted a logistic function to the RT data obtained from the training session: $y=1 /\left(1+10^{-\left(\alpha_{2}^{*} x+\beta_{2}\right)}\right)$, where $y$ is the RT for a given stimulus $x$ ( $x$ is again the spatial phase coherence level), and $\alpha_{2}$ and $\beta_{2}$ are free parameters of the function that are fitted to the RT data using the function FitLogistic.m. With the fitted parameters we computed the chronometric function (ComputeLogistic.m) to obtain the expected mean RTs for the above chosen individual spatial coherence levels.

Pictures at the three $\mathrm{COH}$ levels were shown in the following experimental sessions and the corresponding mean RT was used to determine the speed criterion in the speed condition of the experimental sessions.

$\leftarrow$

$2.20, p=0.14)$. Interactions between SAT and $\mathrm{COH}$ were significant only for $\mathrm{RTs}\left(F_{(2,18)}=9.01\right.$, $p=0.0019)$ indicating that with decreasing $\mathrm{COH}, \mathrm{RT}$ differences between speed and accuracy conditions were larger. Because the interaction is ordinal, main effects could also be interpreted. Error bars present \pm 1 SEM. 
Table 1. Average values and statistics (two-way repeated-measures ANOVA) for behavioral and diffusion model data

\begin{tabular}{|c|c|c|c|c|c|c|c|c|c|c|c|c|}
\hline & \multicolumn{6}{|c|}{ Experimental conditions } & \multirow{2}{*}{\multicolumn{2}{|c|}{ Main effect SAT }} & \multirow{2}{*}{\multicolumn{2}{|c|}{ Main effect $\mathrm{COH}$}} & \multirow{2}{*}{\multicolumn{2}{|c|}{ Interaction SAT $\times \mathrm{COH}$}} \\
\hline & \multicolumn{3}{|l|}{ Speed } & \multicolumn{3}{|l|}{ Accuracy } & & & & & & \\
\hline & High COH & Medium $\mathrm{COH}$ & Low $\mathrm{COH}$ & High $\mathrm{COH}$ & Medium $\mathrm{COH}$ & Low $\mathrm{COH}$ & $F_{(1,9)}$ & $p$ & $F_{(2,18)}$ & $p$ & $F_{(2,18)}$ & $p$ \\
\hline Reaction time & 435 & 456 & 472 & 496 & 530 & 570 & 21.42 & 0.0012 & 30.52 & $<0.001$ & 9.01 & 0.0019 \\
\hline$\%$ correct & 0.88 & 0.77 & 0.58 & 0.97 & 0.86 & 0.64 & 9.89 & 0.012 & 104.43 & $<0.001$ & 2.28 & 0.13 \\
\hline Boundary & 0.064 & 0.053 & 0.052 & 0.15 & 0.10 & 0.081 & 10.35 & 0.011 & 4.89 & 0.020 & 1.80 & 0.19 \\
\hline Drift rate & 0.99 & 0.24 & 0.04 & 1.37 & 0.80 & 0.16 & 1.66 & 0.23 & 11.27 & $<0.001$ & 0.28 & 0.76 \\
\hline Nondecision time & 418 & 413 & 415 & 409 & 440 & 447 & 2.57 & 0.14 & 2.20 & 0.14 & 3.08 & 0.071 \\
\hline
\end{tabular}

Average values and statistics (two-way repeated-measures ANOVA) are reported for reaction times, percentage of correct responses, boundary, drift rate, and nondecision time.

In the experimental sessions (day 2 and 3 ), images of faces or houses at the three chosen $\mathrm{COH}$ levels were presented in random order. Subjects were further instructed on each individual trial to respond either as fast or as accurately as possible (SAT). Thus, stimuli were presented in a $2 \times 3 \times 2$ factorial design (face and house stimuli; three $\mathrm{COH}$ levels; speed or accuracy instruction). Two identical experimental sessions were conducted on successive days to gain a sufficient number of trials for each of the conditions. We restricted the analysis for this report to the face stimuli for two reasons. First, face and house stimuli entail different sensory processes (Kanwisher et al., 1997; Haxby et al., 2000). We used the face stimuli to assure the occurrence of the M170, a component involved in sensory processing that is extensively described in the literature (Swithenby et al., 1998; Liu et al., 2000). Second, motor processing for the two types of stimuli differed because subjects were asked to press a left button with their left thumb when they perceived a face, and a right button with their right thumb when they perceived a house.

At the beginning of each trial, a colored cross was shown for $500 \mathrm{~ms}$ (red for speed, green for accuracy instruction) (Fig. 1A). This task instruction cue was immediately followed by a white fixation cross (presentation time varying from 1000-1600 ms) which signaled subjects not to blink or move their eyes. Subsequently, a picture of a face or a house was presented for $100 \mathrm{~ms}$ in one of three possible $\mathrm{COH}$ levels matching individual performance levels of 95,82 , and $70 \%$ correctness. Under accuracy instructions, subjects had maximal $3000 \mathrm{~ms}$ to respond (otherwise the response was not counted) and heard an unpleasant low-frequency tone after an incorrect decision. Under speed instructions, they had maximal $1300 \mathrm{~ms}$ to respond, and negative feedback was given if their RT was slower than their expected mean RT corresponding to the particular $\mathrm{COH}$ level presented, regardless of the correctness of their decision. No positive feedback was given in the experimental sessions. Button press and feedback, if given, were followed by a $1000 \mathrm{~ms}$ break before the next trial started (Fig. $1 A$ ).

One block consisted of 36 trials, 18 face and 18 house trials with six (training) or three (experiment) $\mathrm{COH}$ levels in random order. Speed and accuracy instructions could change every two blocks in random order. At the end of each block, the subjects received feedback about their mean RT and accuracy (training and experiment). The training session consisted of 24 blocks resulting in 864 trials, 72 per condition. Each experimental session consisted of 24 blocks, 12 with accuracy and 12 with speed instructions. The two experimental sessions resulted in 144 trials per condition.
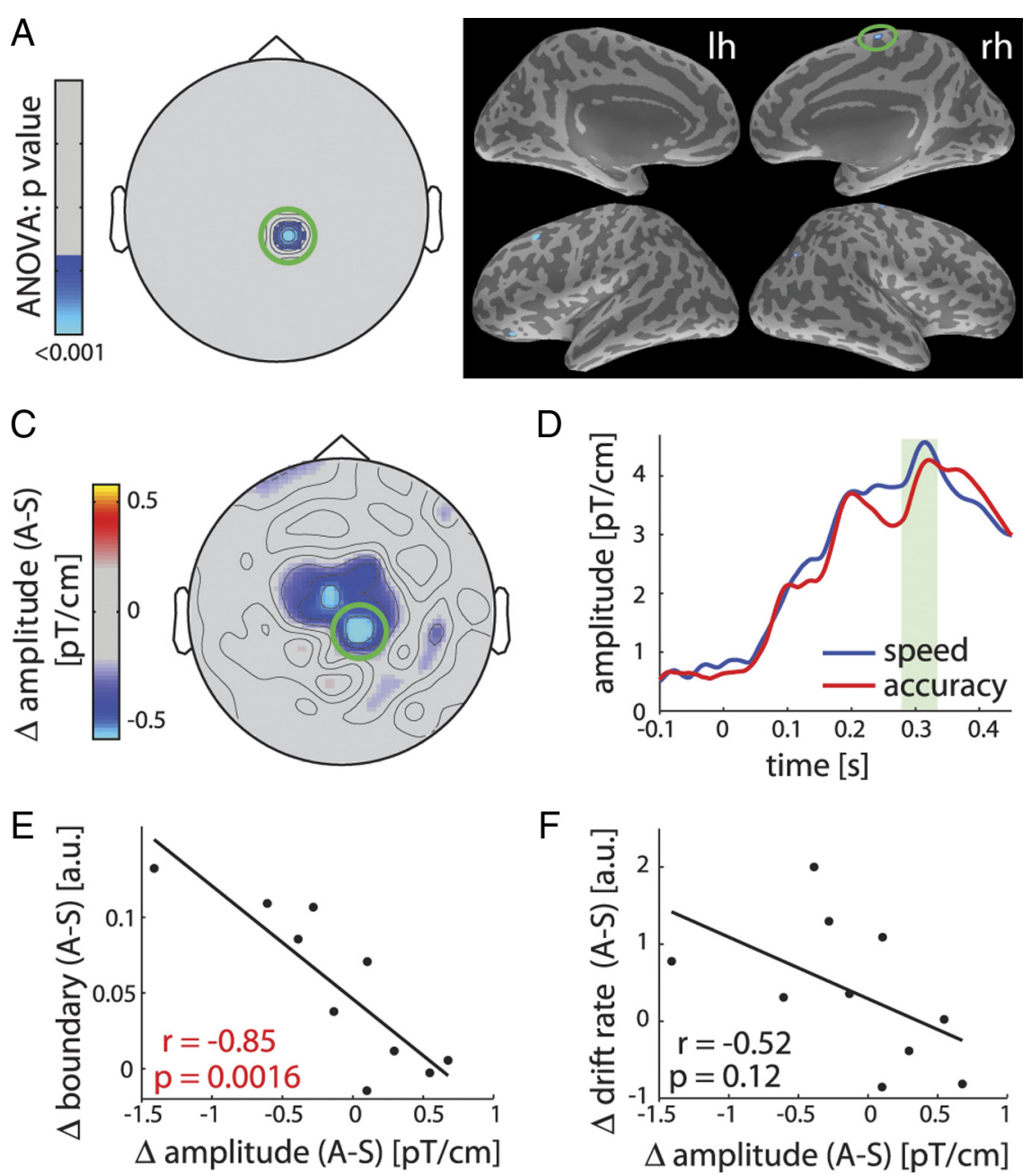

Figure 2. SAT effect between 275 and $325 \mathrm{~ms}$ after stimulus onset in the right SMA negatively correlated with the boundary. $\boldsymbol{A}$, Significant SAT effect on the MEG amplitude during the epoch of interest in a sensor located mediofrontally above the right hemisphere $\left(F_{(1,9)}=23.21, p<0.001\right)$. B S Significant SAT effect on the MEG amplitude during the epoch of interest was localized in the right SMA, BA6, central MNI coordinates $x, 6 ; y,-13 ; z, 71\left(F_{(1,9)}=25.64, p<0.001\right)$. C Grand averaged difference topography (accuracy-speed) of the MEG activity during the epoch of interest. $\boldsymbol{D}$, Time course of this sensor of interest in speed and accuracy instructions. Stimulus onset at $0 \mathrm{~ms}$. The epoch of interest is highlighted by a green box showing higher activity under speed than accuracy conditions. $\boldsymbol{E}$, Highly significant negative correlation during the epoch of interest between the differential (accuracy-speed) sensor space MEG amplitude and the differential (accuracy-speed) boundary ( $r=-0.85, p=0.0016)$, one correlation point per subject. $\boldsymbol{F}$, No significant correlation between the differential MEG amplitude and the differential drift rate $(r=-0.52, p=0.12)$.

Before the training session and experimental sessions, subjects completed 100 practice trials with a similar procedure to assure consistent performance during the training and experimental sessions.

\section{Data acquisition}

Behavioral and electrophysiological data were recorded simultaneously in an electromagnetically shielded room (Vacuumschmelze) using the Presentation software (Neurobehavioral Systems Inc.) and Neuromag software (Elekta 
A

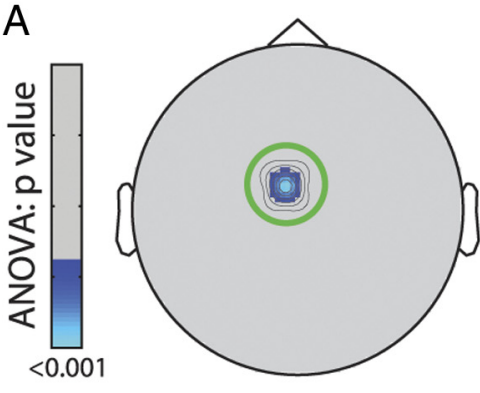

C

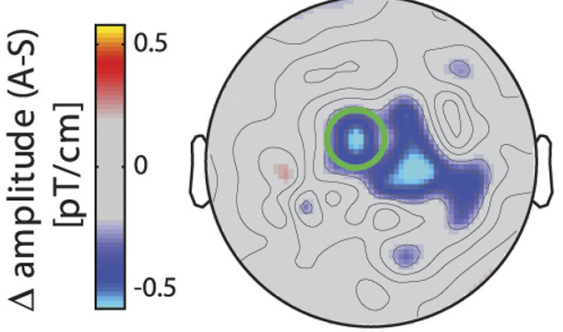

E

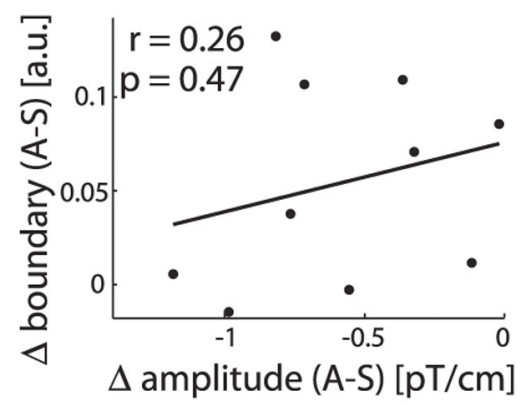

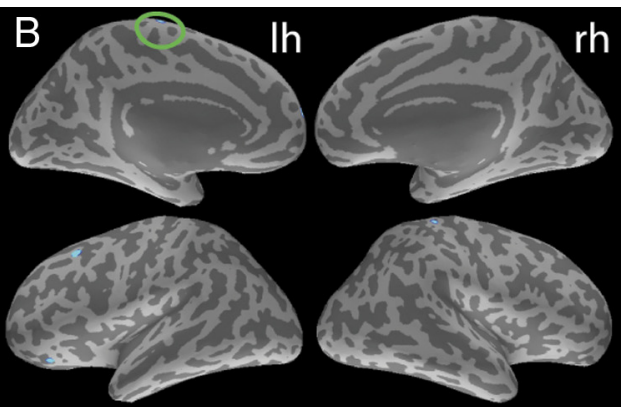

D

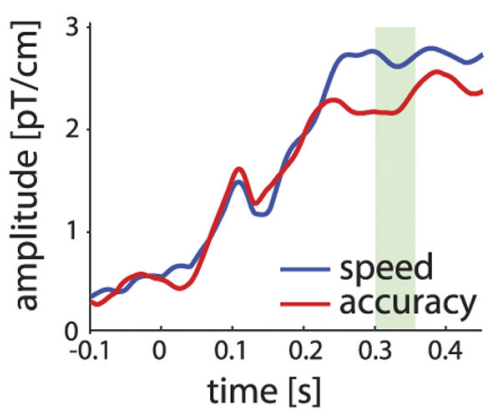

F

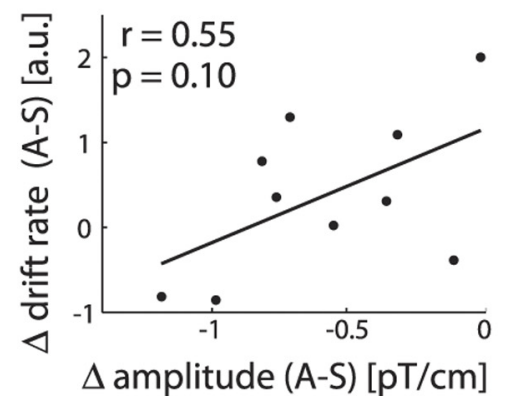

Figure 3. SAT effect between 300 and $350 \mathrm{~ms}$ after stimulus onset in the left SMA not correlated with the boundary. $\boldsymbol{A}$, Significant SAT effect on the MEG amplitude during the epoch of interest in a sensor located mediofrontally above the left hemisphere $\left(F_{(1,9)}=24.04, p<0.001\right)$. B , The SAT effect on the MEG amplitude during the epoch of interest was localized in the left SMA, BA6, central MNI coordinates $x,-2 ; y,-15 ; z, 71\left(F_{(1,9)}=23.72, p<0.001\right)$. C, Grand averaged difference topography (accuracy-speed) during the epoch of interest. $\boldsymbol{D}$, Time course of this sensor of interest under speed and accuracy instructions. Stimulus onset at $0 \mathrm{~ms}$. The epoch of interest is highlighted by a green box showing higher activity under speed than accuracy instructions. $\boldsymbol{E}$, Differential (accuracy-speed) sensor space MEG amplitude and boundary were not significantly correlated ( $r=$ $-0.26, p=0.47$ ). $\boldsymbol{F}$, Likewise, differential (accuracy-speed) sensor space MEG amplitude and drift rate were not significantly correlated $(r=0.55, p=0.10)$.

Neuromag). MEG data were acquired with a 306 channel Vectorview MEG (Elekta Neuromag), 204 planar gradiometers, and 102 magnetometers at 102 locations above the subject's head. Magnetic fields were sampled at 700 $\mathrm{Hz}$ with an online bandpass filter of $0.03-230 \mathrm{~Hz}$. Two pairs of electrodes recorded vertical and horizontal bipolar electrooculograms. Subjects' head positions were recorded by five continuous head position indicator (cHPI) coils (two placed on the auricles, three on the forehead). These coils emit small magnetic fields that are measured by the MEG sensors and can be used to locate each coil to correct for potential movements of the subject's head every $200 \mathrm{~ms}$.

\section{Behavioral data analysis}

Mean RT and accuracy were analyzed, and separate two-way repeatedmeasures ANOVAs with COH (high vs medium vs low) and SAT (speed vs accuracy) were calculated to test for statistically significant differences between the experimental conditions. Outliers above 2 SDs from the mean were removed from the analysis (a total of $4.23 \%$ ). This strict criterion was chosen because we applied no additional outlier treatment for the diffusion model analysis (see next paragraph).
Diffusion model analysis

The diffusion model assumes that decisions in two-alternative forced-choice tasks are made by a stochastic process that accumulates sensory information over time from a starting point toward one of two response thresholds (boundaries). Once either of the boundaries is reached, a response is initiated. From the frequency of correct responses and the RT distributions for correct and error responses, several parameters for each of the experimental conditions are estimated: the starting point, the accumulation rate of the stimulus information (drift rate), the amount of information accumulated to reach the boundary, the portion of RT taken by nondecision components such as encoding and response execution, as well as the amount of variability across trials in each of these parameters (Ratcliff and McKoon, 2008). Note that in the Ratcliff diffusion model, shifts in starting point are equivalent to shifts in the boundary (Ratcliff, 2006), resulting in changes in the distance between starting point and boundary.

In the context of the diffusion model, a classical interpretation is that changes in the emphasis of either speed or accuracy of a decision (here, SAT) are instantiated by changes in boundary whereas changes in the quality of the stimulus information (here, $\mathrm{COH}$ ) are reflected by changes in drift rate (Ratcliff and McKoon, 2008). Furthermore, Rinkenauer et al. (2004) found that SAT also affects nondecision components of the decision process; more precisely, the speed instruction might lead to a decrease in encoding time. Therefore, calculations of the boundary, the drift rate, and the nondecision time were of special interest in this experiment.

We used the diffusion model analysis toolbox (DMAT, http://ppw.kuleuven.be/okp/ dmatoolbox/) for parameter estimation. Here, a multinominal likelihood function expressing the probability of observing a certain proportion of responses in five RT bins was maximized for good parameter estimations (Vandekerckhove and Tuerlinckx, 2007). Four models were nested in the following order: (1) All parameters were restricted to be equal across the conditions, (2) the boundary was free to vary across conditions, (3) additionally, the drift rate was free to vary across conditions, (4) additionally, the nondecision time was free to vary across conditions. Nesting is advantageous because each set of parameter estimates is used as an initial guess for the next model, leading to an increase in efficiency, especially when choosing more restrictive models first and less restrictive later (Vandekerckhove and Tuerlinckx, 2008). To choose the best model for each subject, we used the Bayesian Information Criterion (BIC) and a likelihood ratio test under the assumption of an approximate $\chi^{2}$ distribution. Changes in the order of parameter release revealed identical results. Outliers were defined as RTs above $2 \mathrm{SD}$ from the mean and removed before model calculations. After outliers were removed, the shortest RT was $>250 \mathrm{~ms}$ (i.e., no fast guesses). No additional outlier treatment was applied.

Separate two-way repeated-measures ANOVAs with the factors SAT and $\mathrm{COH}$ were calculated for the boundary, the drift rate, and the nondecision time, respectively, to test for statistical significance between the experimental conditions. 


\section{MEG data analysis}

Sensor-space data were preprocessed with the Neuromag software (Elekta Neuromag). Measured magnetic fields were corrected for head movements using the information from the cHPI coils. Thereby, Maxfilter was applied to interpolate the measured data on a default-gradiometer position and to suppress external interferences (Taulu et al., 2004, 2005). Bad channels were excluded after visual inspection and also interpolated by the Maxfilter procedure. All further analysis was conducted using the MNE software provided by M. Hämäläinen, Massachusetts General Hospital, Boston, MA (http://www.nmr. mgh.harvard.edu/martinos/userInfo/data/ sofMNE.php).

Continuous MEG recordings were divided into epochs of $1200 \mathrm{~ms}$, time-locked either to the stimulus ( -200 to $+1000 \mathrm{~ms})$ or the response $(-1000$ to $+200 \mathrm{~ms})$. Epochs were artifact-corrected by excluding changes $>200$ $\mathrm{pT} / \mathrm{m}$ (gradiometers), $4 \mathrm{pT}$ (magnetometers), or $100 \mu \mathrm{V}$ (electrooculogram, EOG). For the response-locked analysis, the same epochs were used as for the stimulus-locked analysis. Epochs were low-pass filtered with $25 \mathrm{~Hz}$ and averaged separately per condition. The interval from -200 to $0 \mathrm{~ms}$ before the stimulus onset was used as a baseline for stimulus- and response-locked data. Data were averaged across subjects. Two subjects had to be excluded from the MEG analysis due to technical problems. To detect components of interest, MEG amplitudes were averaged using a sliding time window of $50 \mathrm{~ms}$ from 0 to $400 \mathrm{~ms}$ for the stimulus-locked analysis, and from -400 to 0 $\mathrm{ms}$ for the response-locked analysis. The time $400 \mathrm{~ms}$ was chosen because this was the fastest mean RT (speed, high sensory evidence). The vector magnitude of each pair of orthogonal planar gradiometers was computed reflecting the magnetic field strength regardless of the orientation of the gradient. The resultant magnitude displays maximal activity in the sensors located directly above the source (Bastiaansen and Knösche, 2000).

Only correct trials, i.e., when subjects correctly identified the stimulus category regard-

less of the given feedback, were included in the analysis. The analysis was restricted to face trials (for explanation, see Materials and Methods section describing the task).

To test for statistical significance between the experimental conditions, we calculated two-way repeated-measures ANOVAs with the factors SAT and $\mathrm{COH}$. The significance threshold was set to the conservative value of 0.001 . We did not apply a standard correction of the multiplecomparison problem, e.g., Bonferroni correction, since this assumes independence of the data which is not the case either in the temporal or in the spatial domain.

\section{sLORETA source analysis}

Cortical reconstruction. Individual T1-weighted MRI images were recorded with a 3T MRI scanner (Magnetom Trio, Siemens AG). Individual cortical surfaces were reconstructed with the Freesurfer software (http://surfer.nmr.mgh.harvard.edu/) by cutting off tissue which did not correspond to the brain (Ségonne et al., 2004), segmenting white matter (Fischl et al., 2002), estimating the boundary between gray and white matter, and tessellating the cortical surface for each hemisphere (Dale et al., 1999). The MRI coordinate system was transformed into the MEG coordinate system by aligning the individual white matter surface with
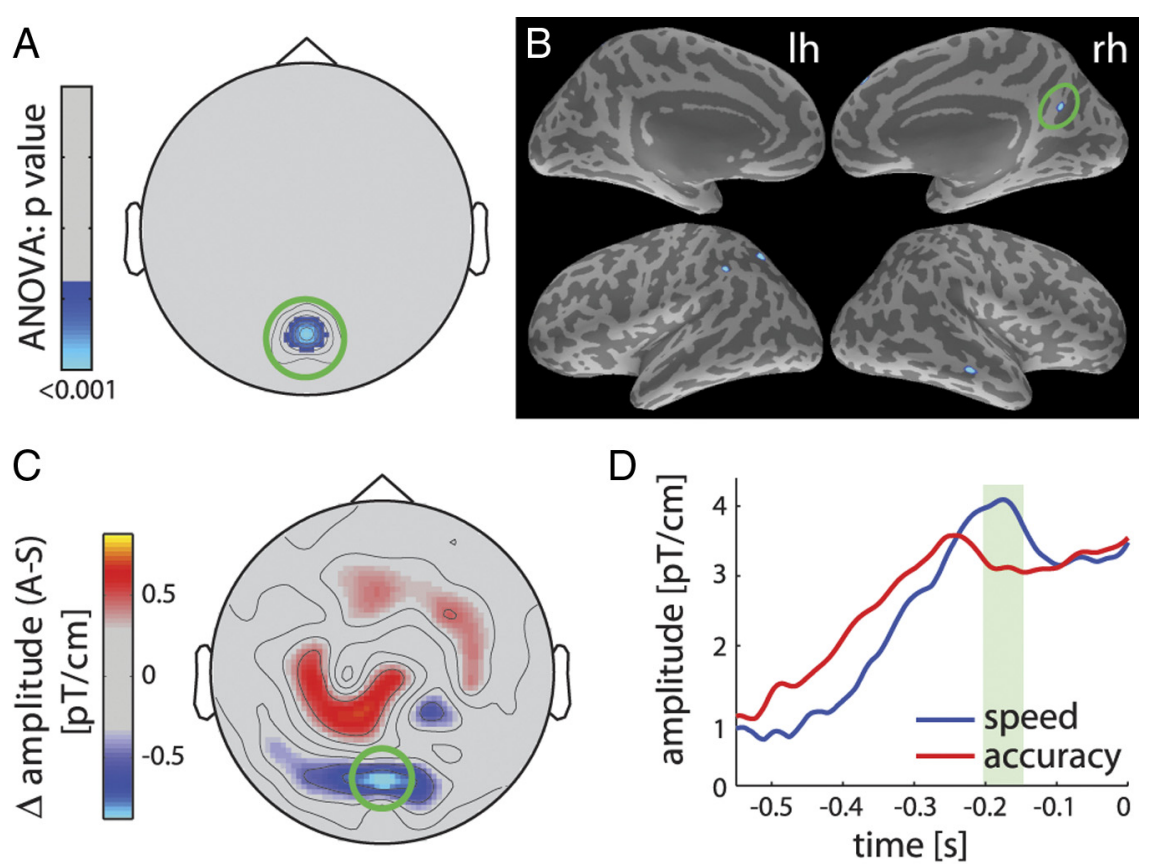

$\mathrm{F}$

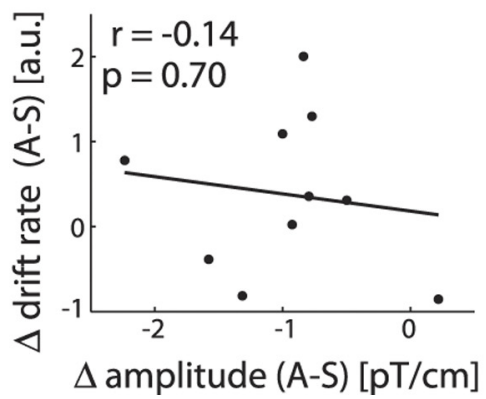

Figure 4. SAT effect between 200 and 150 ms before the response in the medial precuneus not correlated with the boundary. $A$, Significant SAT effect on the MEG amplitude during the epoch of interest in a right parietal sensor $\left(F_{(1,9)}=23.59, p<0.001\right)$. , significant SAT effect on the MEG amplitude during the epoch of interest was localized in the right medial precuneus, BA31, ms. The significant correlation between differential (accuracy-speed) MEG activity and boundary $(r=-0.33, p=0.35)$. $\boldsymbol{F}$, Likewise, no significant correlation between differential (accuracy-speed) MEG activity and boundary $(r=-0.14, p=0.70)$.

the cHPI coil positions and $\sim 40$ additional points on the head surface recorded with a Polhemus FASTRAK 3D digitizer.

Source reconstruction. As a volume conductor, we used individual boundary element models with one compartment, namely the inner skull surface of the above mentioned reconstructed individual cortical surface. A single compartment volume conductor is widely considered to be sufficient to model MEG data (Hämäläinen and Sarvas, 1989). Individual white matter surfaces were estimated digitally by $\sim 150,000$ vertices for each hemisphere. Source spaces were derived by downsampling these vertices to $\sim 5000 \mathrm{di}-$ poles, resulting in $\sim 1$ dipole in $10 \mathrm{~mm}^{2}$. For the inverse solution, the orientation of each dipole was restricted to be almost orthogonal to the cortical surface (loose factor of 0.2). The individual brain activity was computed with the sLORETA method (Pascual-Marqui, 2002; Pascual-Marqui et al., 2002). To average across subjects, the individual cortical representations were interpolated to the inflated cortical surface of one subject and subsequently averaged (Fischl et al., 1999a,b).

For source analysis, we adjusted the time windows to the epochs where significant differences were found in sensor space. To provide statistical maps of the source results, we calculated whole-brain (20,484 vertex locations) two-way repeated-measures ANOVAs for the stimulus- and response-locked analysis with the factors SAT and $\mathrm{COH}$ and a significance 

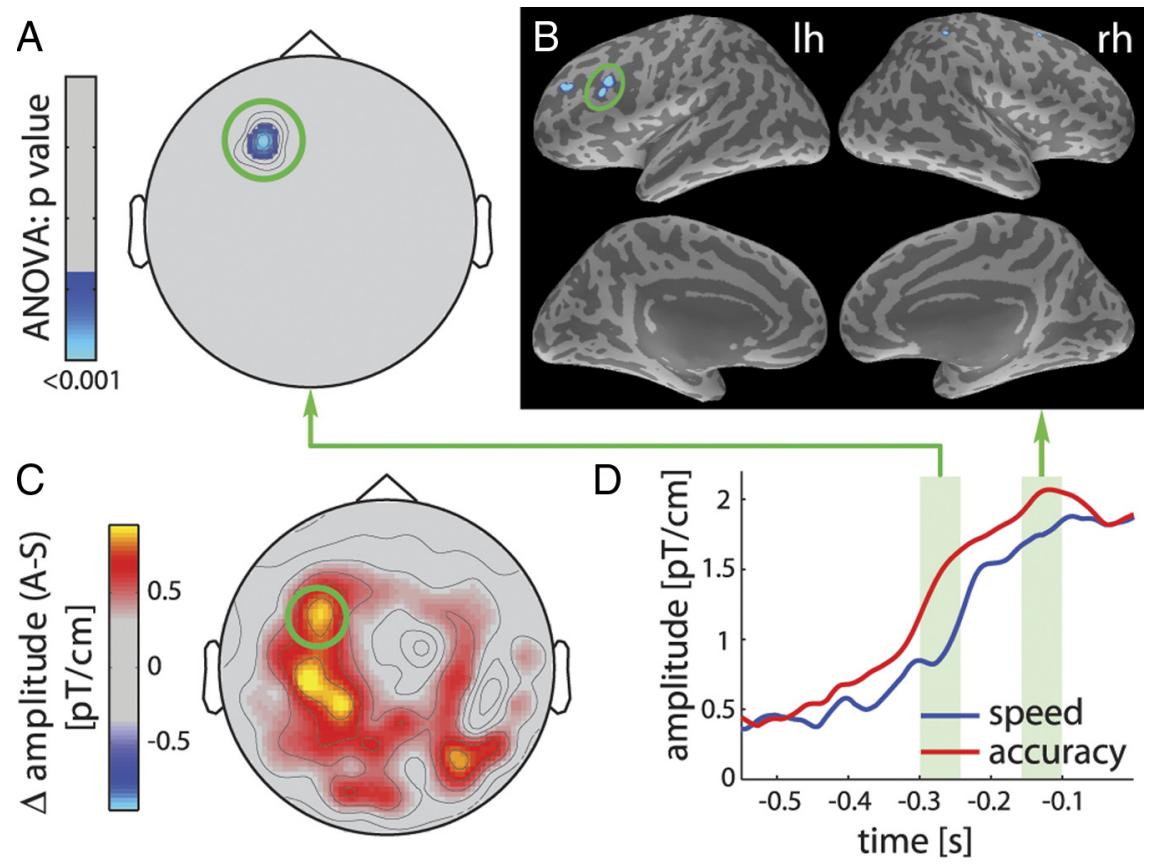

$\mathrm{E}$

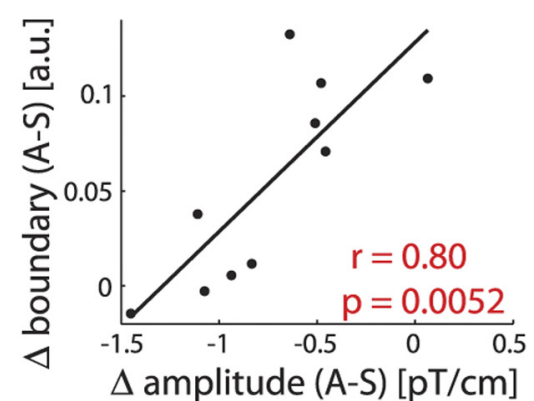

$\mathrm{F}$

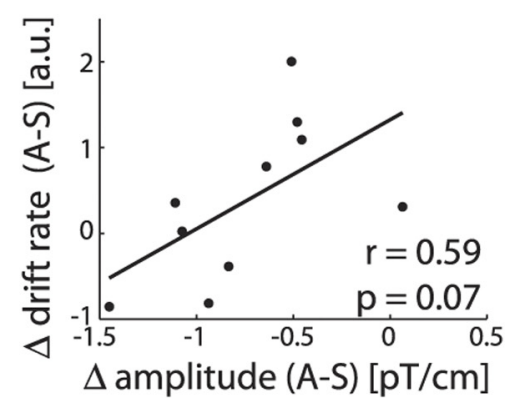

Figure 5. SAT effects between 300 and $250 \mathrm{~ms}$ (early epoch of interest) and between 150 and $100 \mathrm{~ms}$ (late epoch of interest) before the response in the left DLPFC, middle frontal gyrus, BA9. Early differential (accuracy-speed) activity positively correlated with the boundary. $A$, Significant SAT effect on the MEG amplitude during the early epoch of interest in a left laterofrontal sensor $\left(F_{(1,9)}=22.89, p<0.001\right)$. B S Significant SAT effect on the MEG amplitude in the late epoch of interest in the source space $\left(F_{(1,9)}=\right.$ $25.49, p<0.001)$. Early and late left frontal SAT effects were located in the left DLPFC in the middle frontal gyrus, BA9, central Talairach coordinates $x,-41 ; y, 15 ; z, 26$. C, Grand averaged difference topography (accuracy-speed) during the early epoch of interest. $\boldsymbol{D}$, Time course of this sensor of interest under speed and accuracy instructions showing that activity was always higher under accuracy than speed conditions (but see Results for details). Response at 0 ms. Early and late epochs of interest are highlighted by green boxes. $\boldsymbol{E}$, Highly positive correlation between differential (accuracy-speed) sensor space MEG activity and boundary $(r=0.80, p=0.0052)$. $\boldsymbol{F}$, No significant correlation between MEG activity and drift rate $(r=0.59, p=0.07)$.

threshold of 0.001 . We labeled regions of interest by calculating cytoarchitectonic probabilities at defined MNI (Montreal Neurological Institute) coordinates using the SPM8 Anatomy Toolbox, current version 1.7b (http:// www.fz-juelich.de/inm/inm-1/spm_anatomy_toolbox). While some anatomical probability maps are very detailed, e.g., for motor cortices, maps for prefrontal regions are provided for Brodmann area (BA) 44 and BA45 only (Brodmann, 1909). For labeling of prefrontal regions we therefore used the Talairach Applet (http://www.talairach.org/daemon.html). It labels coordinates in the standardized Talairach space according to the atlas of Talairach and Tournoux (1988). The Talairach Applet also provides structural probability maps but currently integrates fewer structures than the SPM8 Anatomy Toolbox.

\section{Prediction of diffusion model parameters from MEG data}

To investigate whether the neural activity recorded with MEG could predict diffusion model parameters calculated from behavioral data, we correlated the amplitudes of the sensor space MEG components with the diffusion model parameters for each individual. Because SAT showed a significant effect on the boundary (see Results), we correlated the differential (accuracyspeed) MEG components showing an SAT effect with the differential (accu- racy-speed) boundary. Because $\mathrm{COH}$ had a significant effect on drift rate and boundary (see Results), we correlated the differential (high-low $\mathrm{COH}) \mathrm{MEG}$ components showing a $\mathrm{COH}$ effect with the differential (high-low $\mathrm{COH}$ ) drift rate and the differential (high-low $\mathrm{COH}$ ) boundary. For consistent description of the data, we also correlated the differential (accuracy-speed) MEG components showing an SAT effect with the differential (accuracy-speed) drift rate.

\section{Results}

Data are reported for 10 subjects who indicated whether they saw the picture of a face or a house by pressing the left button with their left thumb when they perceived a face and the right button with their right thumb when they perceived a house (Fig. $1 A)$. In different blocks they were cued to respond either as fast or as accurately as possible (SAT). Sensory evidence of the stimuli $(\mathrm{COH})$ was manipulated by changing the phase coherence of the pictures according to individual performance of 95,82 , and $70 \%$. In the present report, the analysis was restricted to face trials (see task section of Materials and Methods for explanation).

\section{Behavioral data}

In the speed compared with the accuracy condition, subjects showed significantly faster RTs $\left(F_{(1,9)}=21.42, p=0.0012\right)$ (Fig. $1 B$ ) and committed more errors $\left(F_{(1,9)}=9.89, p=0.012\right.$ ) (Fig. $\left.1 C\right)$. With decreasing $\mathrm{COH}$ subjects had slower RTs $\left(F_{(2,18)}=30.52, p<0.001\right)($ Fig. $1 B)$ and lower percentage correct $\left(F_{(2,18)}=104.43\right.$, $p<0.001$ ) (Fig. 1C). Interactions between SAT and COH were significant for RT $\left(F_{(1,9)}\right.$ $=9.01, p=0.0019)$ indicating that with decreasing $\mathrm{COH}$ RT differences between speed and accuracy conditions were larger. Because the interaction was ordinal, main effects could also be interpreted. The interaction was not significant for percentage correct $\left(F_{(2,18)}=2.28, p=0.13\right)$. Average values per condition (speed versus accuracy and high versus medium versus low coherence) and statistics are presented in Table 1.

\section{Diffusion model data}

According to the classical interpretation of the diffusion model, changes in the speed-accuracy tradeoff affect the boundary while changes in the quality of sensory information $(\mathrm{COH})$ primarily affect the drift rate (Ratcliff and McKoon, 2008). Additionally, Rinkenauer et al. (2004) found the nondecision time to be shorter under speed than accuracy instructions. Therefore, these three parameters were of special interest here. To calculate the diffusion model, four models were nested in the following order: (1) all parameters were restricted to be equal across the conditions, (2) the boundary was free to vary across conditions, (3) additionally, the drift rate was free to vary across conditions, (4) and the nondecision time was free to vary across conditions (see Materials and Methods). For all subjects, the model fit improved signif- 
icantly when leaving boundary and drift rate free (model 3) compared with when only leaving the boundary free (model 2). For five of 10 subjects, BIC values revealed a significantly better fit when leaving additionally the nondecision time free (model 4). Accordingly, we chose the "best fit" model for each subject: for five subjects only boundary and drift rate were allowed to vary freely across conditions whereas the nondecision time was restricted to be equal across conditions and for the other five subjects all three parameters were allowed to vary freely across conditions.

As expected, under speed compared with accuracy conditions the boundary was significantly lower $\left(F_{(1,9)}=10.35, p=\right.$ 0.011 ) (Fig. 1D) but the drift rate did not differ significantly $\left(F_{(1,9)}=1.66, p=0.23\right)$ (Fig. 1E). Speed versus accuracy conditions also had no effect on the nondecision time $\left(F_{(1,9)}=2.57, p=0.14\right)$. With decreasing $\mathrm{COH}$, the drift rate decreased $\left(F_{(2,18)}=11.27, p<0.001\right)$ (Fig. $\left.1 E\right)$ and the nondecision time did not differ significantly $\left(F_{(2,18)}=2.20, p=0.14\right)$, also meeting the expectations. Surprisingly, the boundary also showed a significant $\mathrm{COH}$ effect $\left(F_{(2,18)}=4.89, p<0.020\right)$ (Fig. 1D). Interactions between SAT and $\mathrm{COH}$ were not significant for any of the parameters (boundary: $F_{(2,18)}=1.80, p=$ 0.19 ; drift rate: $F_{(2,18)}=0.28, p=0.76$; nondecision time: $F_{(2,18)}=3.08, p=$ $0.071)$. For the average values as well as statistics of the diffusion model parameters we refer the reader to Table 1.

\section{MEG data analysis, source}

reconstruction, and correlation of the event-related fields with diffusion model parameters

All MEG sensor data were baseline-cor-

rected to the period before stimulus onset ( -200 to $0 \mathrm{~ms}$ ) and MEG amplitudes were averaged using a sliding time window of $50 \mathrm{~ms}$. The sensor data show the combined measurements of orthogonal planar gradiometers, reflecting the absolute strength of stimulus-evoked neural activity with a spatial peak in sensors closely overlying its cortical source (Bastiaansen and Knösche, 2000). To test for statistical significance, we calculated two-way repeated-measures ANOVAs for the stimulus- and response-locked analysis with the factors SAT and $\mathrm{COH}$ and a significance threshold of 0.001 .

To localize these components, we conducted a distributed source analysis (sLORETA) for the time windows showing significant effects in the sensor space and evaluated significance again using repeated-measures ANOVAs for the stimulus- and response-locked analysis with the factors SAT and $\mathrm{COH}$ and a significance threshold of 0.001 .

We correlated the amplitudes of the sensor space MEG components with the diffusion model parameters for each individual to investigate whether the neural activity recorded with MEG could predict diffusion model parameters calculated from behavioral data. Because SAT showed a significant effect on the boundary, we cor-
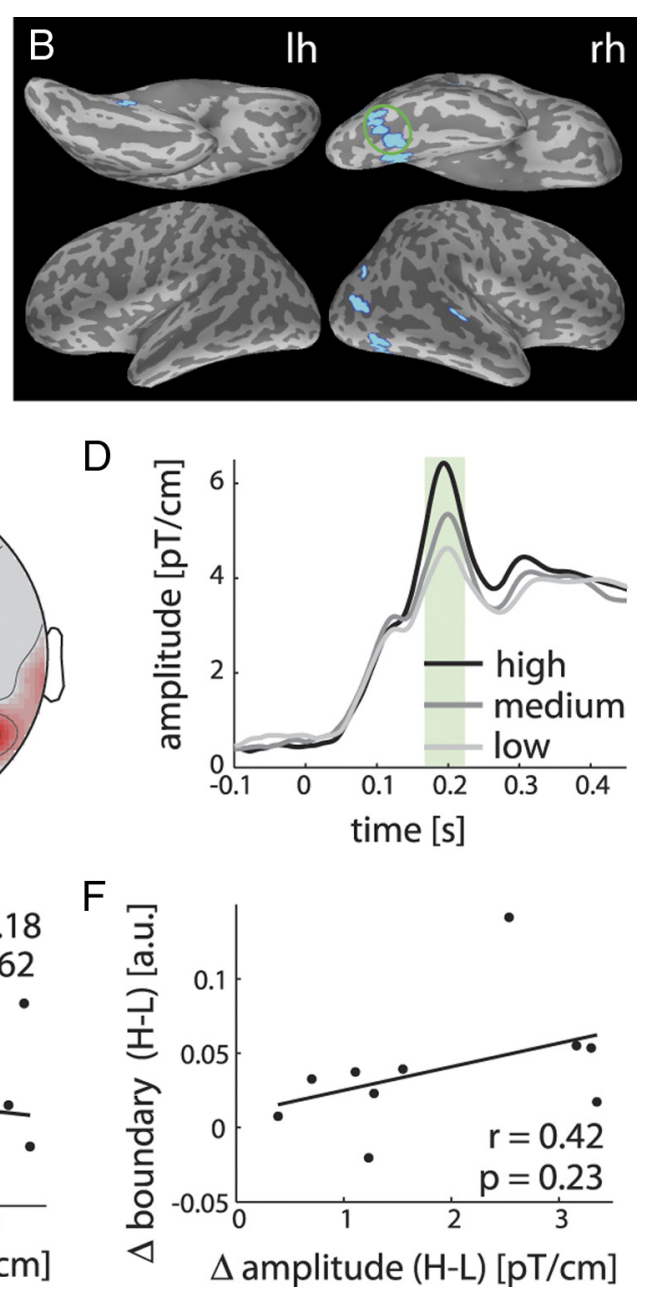

$\Delta$ amplitude $(\mathrm{H}-\mathrm{L})[\mathrm{pT} / \mathrm{cm}]$

Figure 6. $\mathrm{COH}$ effect between 150 and $200 \mathrm{~ms}$ (M170) after stimulus onset in the right fusiform gyrus not correlated with the drift rate. $\boldsymbol{A}$, Significant $\mathrm{COH}$ effect on the MEG amplitude during the epoch of interest in right mediotemporal sensors $\left(F_{(2,18)}=15.36, p<0.001\right)$. in coherence) sensor space MEG amplitude and drift rate were not significantly correlated ( $r=-0.18, p=0.62)$. F, Likewise, differential MEG amplitude and boundary were not significantly correlated $(r=0.42, p=0.23)$.

related the differential (accuracy-speed) MEG components showing an SAT effect with the differential (accuracy-speed) boundary. Because $\mathrm{COH}$ had a significant effect on drift rate and boundary, we correlated the differential (high-low $\mathrm{COH}$ ) MEG components showing a $\mathrm{COH}$ effect with the differential (high-low $\mathrm{COH}$ ) drift rate and the differential (high-low $\mathrm{COH})$ boundary. For consistent description of the data, we also correlated the differential (accuracy-speed) MEG components showing an SAT effect with the differential (accuracy-speed) drift rate.

We identified four MEG components that differed significantly between the speed and the accuracy condition, two in the stimuluslocked and two in the response-locked analysis. Furthermore, five components differed significantly with respect to $\mathrm{COH}$, four in the stimulus-locked and one in the response-locked analysis. No significant interactions between SAT and $\mathrm{COH}$ manipulations were found. In the following sections we describe each of the components, the corresponding source localization results, and its correlation with the appropriate diffusion model parameter(s) in temporal order, starting with significant SAT effects. 
A
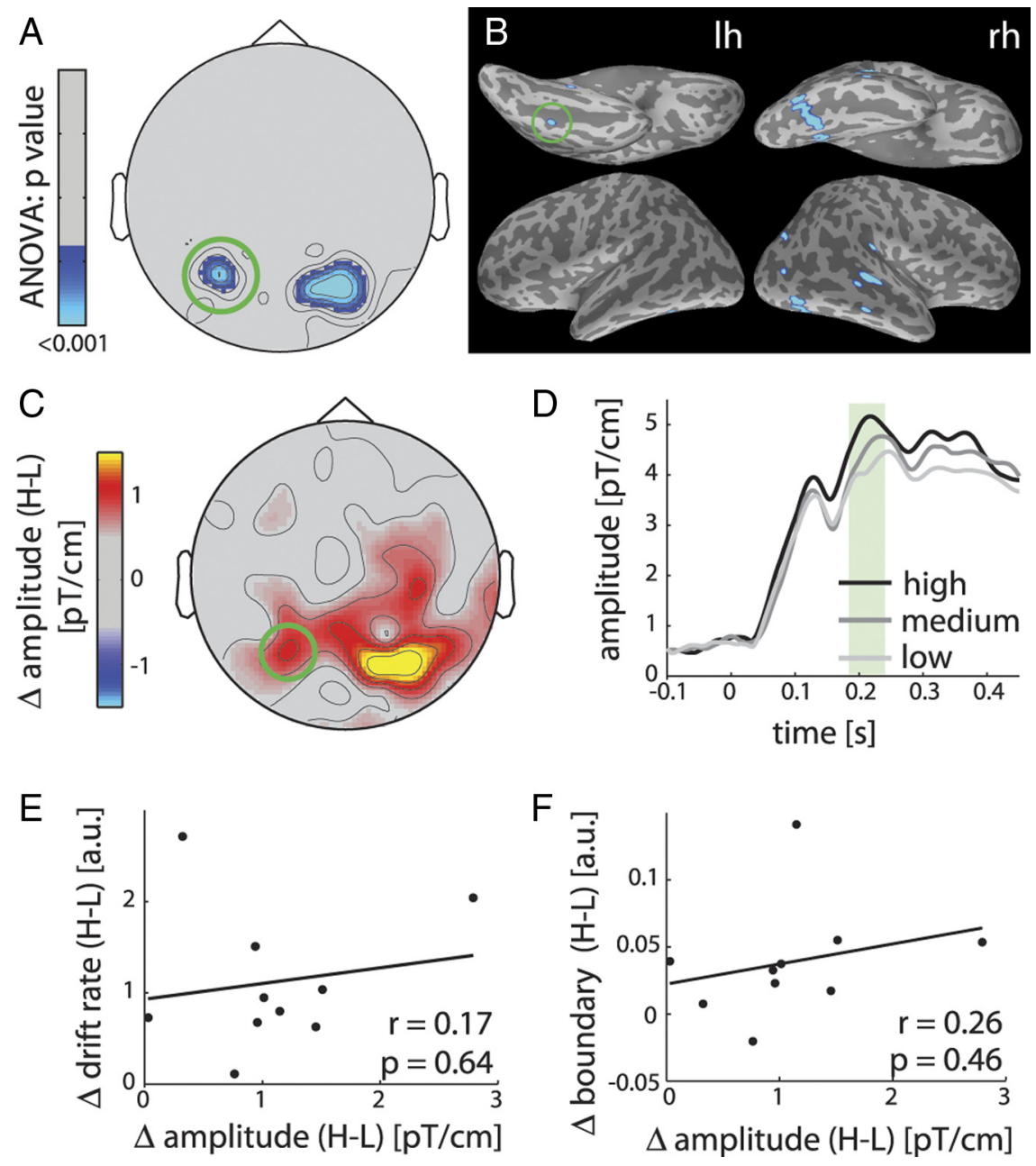

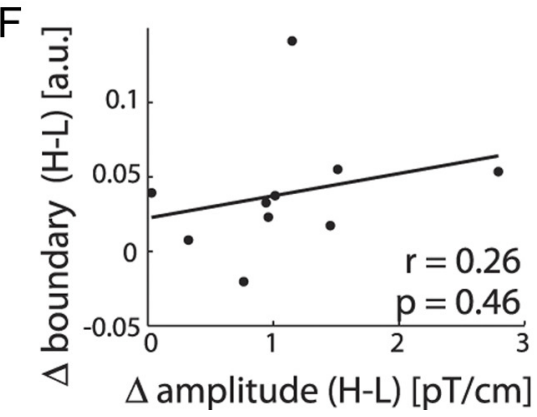

Figure 7. Significant $\mathrm{COH}$ effect between 185 and $235 \mathrm{~ms}$ (M170) after the stimulus onset in the left fusiform gyrus not correlated with the drift rate. $\boldsymbol{A}$, Significant $\mathrm{COH}$ effect on the MEG amplitude during the epoch of interest in left mediotemporal sensors $\left(F_{(2,18)}=13.03, p<0.001\right)$, together with the aforementioned $\mathrm{COH}$ effect in right mediotemporal sensors. $\boldsymbol{B}$, Significant $\mathrm{COH}$ effect on the MEG amplitude during the epoch of interest in the source space $\left(F_{(2,18)}=12.87, p<0.001\right)$. It was located in the left fusiform gyrus, central MNI coordinates $x,-41 ; y,-56 ; z,-14$. C, Grand averaged difference topography (high-low coherence) during the epoch of interest. $\boldsymbol{D}$, Time course of these sensors of interest in response to high, medium, and low $\mathrm{COH}$ stimuli. Stimulus onset at $0 \mathrm{~ms}$. The epoch of interest is highlighted by a green box showing, similar to right fusiform gyrus, decreasing amplitudes with decreasing $\mathrm{COH}$. $\boldsymbol{E}$, Differential sensor space MEG amplitude and drift rate were not significantly correlated $(r=$ $0.17, p=0.64)$. $\boldsymbol{F}$, Likewise, differential sensor space MEG amplitude and boundary were not significantly correlated $(r=0.26$, $p=0.46)$.

SAT component between 275 and $325 \mathrm{~ms}$ after stimulus onset in the right SMA negatively correlated with the boundary

A right mediofrontal sensor showed a greater MEG amplitude under the speed instruction than the accuracy instruction between 275 and $325 \mathrm{~ms}$ after stimulus onset $\left(F_{(1,9)}=23.21, p<0.001\right)$ (Fig. $\left.2 A\right)$. The sensor with the significant effect was also the one with the greatest activity in the difference topography (accuracy-speed) (Fig. 2C). The time course of activity of this sensor showed higher activity under speed than accuracy instructions between 275 and 325 ms (Fig. 2D). Source localization during the epoch of interest localized the component in the right SMA, BA6, with a probability of $90 \%$ [70-100\%], central MNI coordinates $x, 6 ; y,-13 ; z, 71$. Similar to the results in the sensor space, the activity in the source space showed greater activity under the speed than the accuracy instruction $\left(F_{(1,9)}\right.$ $=25.64, p<0.001$ ) (Fig. $2 B$ ). Difference (accuracy-speed) in the sensor space MEG amplitude and the boundary were highly negatively correlated ( $r=-0.85, p=0.0016)$ (Fig. $2 E$ ). On the contrary, differential MEG activity and drift rate were not significantly correlated $(r=-0.52, p=0.11)$ (Fig. $2 F)$.
SAT component between 300 and $350 \mathrm{~ms}$ after stimulus onset in the left SMA not correlated with the boundary

A left mediofrontal sensor showed a greater MEG amplitude under the speed than the accuracy instruction between 300 and 350 ms after stimulus onset $\left(F_{(1,9)}=24.04, p<\right.$ 0.001 ) (Fig. 3A). The sensor showing the significant effect also showed the greatest activity in the difference topography (accuracyspeed), together with the aforementioned sensor localized in the right SMA (Fig. 3C). The time course of the activity under speed and accuracy instructions of this sensor showed that higher activity levels under speed than accuracy instructions started around $220 \mathrm{~ms}$ and thereafter stayed on these activity levels (Fig. 3D). Source localization during the epoch of interest localized the component in the left paracentral lobule, BA6, with a probability of 70\% [50$80 \%$ ] whereas the probability for the anterior part of the primary motor cortex (BA4a) was $10 \%$ only, central MNI coordinates $x,-2 ; y,-15 ; z, 71$. The medial BA6 includes SMA, pre-SMA, and the supplementary eye field (SEF) (Nachev et al., 2008) but the anatomical coordinates argue against pre-SMA along the anterior-posterior axis (Johansen-Berg et al., 2004) and against SEF along the dorsal-ventral axis (Grosbras et al., 1999). Therefore we label this area left SMA. As for the sensor-space analysis, the activity in the source space showed greater activity under the speed instruction than the accuracy instruction $\left(F_{(1,9)}=23.72, p<0.001\right)$ (Fig. 3B). The differential sensor space MEG activity was not significantly correlated with the boundary $(r=0.26, p=0.47)$ (Fig. $3 E$ ) or the drift rate $(r=0.55, p=0.10)$ (Fig. $3 F)$.

SAT component between 200 and $150 \mathrm{~ms}$ before the response in the right precuneus not correlated with the boundary

A medioparietal sensor showed a greater MEG amplitude under the speed than the accuracy instruction between 200 and $150 \mathrm{~ms}$ before the response $\left(F_{(1,9)}=23.59, p<0.001\right)$ (Fig. $\left.4 A\right)$. The sensor showing the significant effect also showed the greatest activity in the difference topography (accuracy-speed) (Fig. 4C). The time course of the activity under speed and accuracy instructions measured on this sensor showed that greater activity under the speed than the accuracy instruction was most pronounced between 220 and 150 ms before the response (Fig. 4D). Seemingly greater activity under accuracy than speed instructions from $\sim 500$ to 300 ms before the response are likely due to the different mean RTs under speed and accuracy instructions and hence different stimulus-onset times in this response-locked time reference, i.e., reaction time effects. Source localization during the epoch of interest localized this component in the right precuneus (no probability given), BA31, central MNI coordinates $x$, $14 ; y,-59 ; z, 22$. As for the sensor space data, in the source space the precuneus showed greater activity under the speed 
than the accuracy instruction $\left(F_{(1,9)}=\right.$ 29.14, $p<0.001$ ) (Fig. $4 B$ ). The differential sensor space MEG activity was not significantly correlated with the differential boundary $(r=-0.33, p=0.35)$ (Fig. $4 E$ ) or with the differential drift rate $(r=-0.14, p=0.70)$ (Fig. $4 F)$.

SAT component between 300 and $250 \mathrm{~ms}$ in the left DLPFC positively correlated with the boundary

A left lateral prefrontal sensor showed a greater MEG amplitude under the accuracy than the speed instruction between 300 and $250 \mathrm{~ms}$ before the response $\left(F_{(1,9)}\right.$ $=22.89, p<0.001$ ) (Fig. 5A). The sensor showing this effect also showed a high magnitude in the difference topography (accuracy-speed) (Fig. 5C). Source localization during the epoch of interest localized the component in the DLPFC in the left middle frontal gyrus, BA9, central Talairach coordinates $x,-41 ; y, 15 ; z, 26$ (for labeling of prefrontal regions we used the Talairach Applet as the SPM8 Anatomy Toolbox provides maps in prefrontal regions for BA44 and 45 only; cf. Materials and Methods for further details). Similar to the sensor level results, the activity in the source space showed greater activity under the accuracy than the speed instruction $\left(F_{(1,9)}=28.83, p<0.001\right)$. Differential sensor space MEG activity was highly positively correlated with the boundary $(r=0.80, p=$ 0.0052 ) (Fig. 5E), whereas it was not significantly correlated with the drift rate $(r=$ $0.59, p=0.07$ ) (Fig. 5F).

When looking at the time course of this sensor (Fig. 5D), the question might arise if the SAT effect is due to reaction time effects. However, the amplitudes under both instructions reached their maximum between 150 and $100 \mathrm{~ms}$ before response onset, and the amplitude under the accuracy instruction was again significantly higher than under the speed instruction (sensor level: $F_{(1,9)}=6.41, p=0.032$; source space: $F_{(1,9)}=25.49, p<0.001$, cf. Fig. $\left.5 B\right)$. This suggests that the effect derived from reaction time effects as well as true amplitude differences.

\section{COH component 150-200 ms after stimulus onset in the right} fusiform gyrus correlated neither with drift rate nor with boundary Multiple right mediotemporal sensors showed no SAT effect on the MEG amplitude between 150 and $200 \mathrm{~ms}$ after stimulus onset but they showed a significant $\mathrm{COH}$ effect on the amplitude $\left(F_{(2,18)}=15.36, p<0.001\right)$ (Fig. 6A). These sensors also showed the greatest activity in the difference topography (high-low $\mathrm{COH}$ ) (Fig. 6C). The time courses for the different $\mathrm{COH}$ levels in this time interval reveal that with decreasing $\mathrm{COH}$ the amplitude decreased (Fig. 6D). Source localization during the epoch of interest localized the $\mathrm{COH}$ component in the right fusiform gyrus (no probability given), the probability for area hOC4v (V4) was $20 \%$ [10-30\%], central MNI coordinates $x, 31 ; y,-61 ; z,-15$. The right fusiform gyrus showed a significant $\mathrm{COH}$ effect on the amplitude $\left(F_{(2,18)}=17.07, p<0.001\right)$ (Fig. $\left.6 B\right)$. The differential (high-low $\mathrm{COH}$ ) sensor space MEG activity was not correlated with either the differential drift rate $(r=-0.18, p=0.62)$ (Fig. $6 E)$ or the differential boundary $(r=0.42, p=0.23)$ (Fig. $6 F)$.

COH component 185-235 ms after stimulus onset in the left fusiform gyrus correlated neither with the drift rate nor with the boundary

A left mediotemporal sensor showed a significant $\mathrm{COH}$ effect on the MEG amplitude between 185 and $235 \mathrm{~ms}$ after stimulus onset $\left(F_{(2,18)}=13.03, p<0.001\right)($ Fig. $7 A)$. This sensor also showed a high magnitude in the difference topography (high-low $\mathrm{COH}$ ), together with the aforementioned sensors localized in the right fusiform gyrus (Fig. 7C). Like in the right fusiform gyrus, the time courses in this time interval show decreasing amplitudes with decreasing $\mathrm{COH}$ (Fig. 7D). Compared with the right fusiform gyrus, activity was distributed over a smaller area and reached lower peak amplitudes, at least when $\mathrm{COH}$ was high. Source localization during the epoch of interest localized the $\mathrm{COH}$ component in the left fusiform gyrus (no probability given), central 

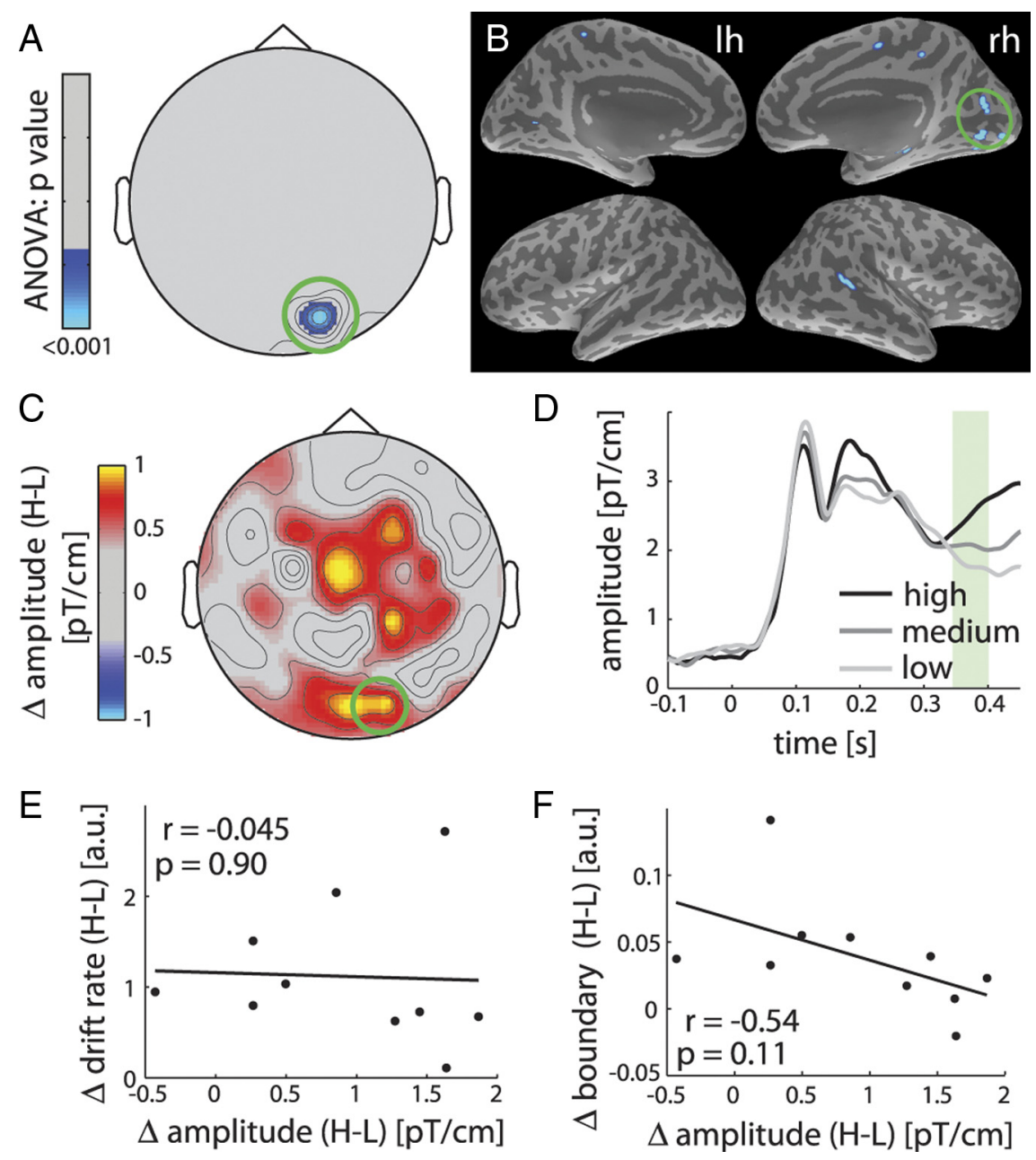

Figure 9. Significant $\mathrm{COH}$ effect between 350 and $400 \mathrm{~ms}$ after the stimulus onset in the right calcarine gyrus not significantly correlated with the drift rate. $\boldsymbol{A}$, Significant $\mathrm{COH}$ effect on the MEG amplitude during the epoch if interest in a right medial parietal sensor $\left(F_{(2,18)}=12.14, p<0.001\right)$. $\boldsymbol{B}$, Significant $\mathrm{COH}$ effect on the MEG amplitude in the source space $\left(F_{(2,18)}=11.70, p<\right.$ 0.001). The $\mathrm{COH}$ effect was located in the right calcarine gyrus, assigned to BA17 with a probability of $90 \%[70-100 \%]$, probability for BA18 was 30\% [20-50\%], central MNI coordinates $x, 8 ; y,-75, z, 7 . C$, Grand averaged difference topography (high-low $\mathrm{COH}$ ) during the epoch of interest. $\boldsymbol{D}$, Time course of this sensor of interest in response to high, medium, and low $\mathrm{COH}$ stimuli showing that with decreasing $\mathrm{COH}$ the amplitude decreased. Stimulus onset at $0 \mathrm{~ms}$. The epoch of interest is highlighted by a green box. $\boldsymbol{E}$, No significant correlation between differential (high-low $\mathrm{COH}$ ) sensor space MEG activity and drift rate $(r=-0.045, p=0.90)$. $\boldsymbol{F}$, Likewise, no significant correlation between differential MEG activity and boundary $(r=-0.54, p=0.11)$.

MNI coordinates $x,-41 ; y,-56 ; z,-14$. As for the sensor space, for the source space data, the left fusiform gyrus showed a significant $\mathrm{COH}$ effect on the amplitude $\left(F_{(2,18)}=12.87, p<0.001\right)$ (Fig. $7 B$ ). The differential (high-low $\mathrm{COH}$ ) sensor space MEG activity was not correlated with either the differential drift rate $(r=0.17, p=0.64)$ (Fig. $7 E)$ or the differential boundary $(r=$ $0.26, p=0.46)($ Fig. $7 F)$.

COH component 285-335 ms after stimulus onset in the right DLPFC correlated with the drift rate but not with the boundary

A right lateral prefrontal sensor showed a significant $\mathrm{COH}$ effect on the MEG amplitude between 285 and $335 \mathrm{~ms}$ after stimulus onset $\left(F_{(2,18)}=10.86, p<0.001\right)$ (Fig. 8A). This sensor also showed high activity in the difference topography (high-low COH) (Fig. 8C). The time courses for the different $\mathrm{COH}$ levels in this time interval show higher amplitudes with increasing $\mathrm{COH}$ (Fig. $8 \mathrm{D}$ ). Source localization during the epoch of interest localized the $\mathrm{COH}$ component in the right middle frontal gyrus, BA9, central Talairach coordinates $x, 38$; $y, 15 ; z, 28$ (for labeling of prefrontal regions we used the Talairach Applet as the SPM8 Anatomy Toolbox provides maps in prefrontal regions for BA44 and 45 only; cf. Materials and Methods for further details). As for the sensor space, for the source space data the right DLPFC showed a significant $\mathrm{COH}$ effect on the amplitude $\left(F_{(2,18)}=12.22, p<\right.$ 0.001 ) (Fig. $8 B$ ). The differential (highlow $\mathrm{COH}$ ) sensor space MEG activity was highly correlated with the differential drift rate $(r=0.83, p=0.0032)$ (Fig. $8 E$ ). It was not significantly correlated with the differential boundary $(r=$ $-0.17, p=0.64$ ) (Fig. $8 F$ ).

\section{COH component 350-400 ms after} stimulus onset in the right calcarine gyrus correlated neither with the drift rate nor with the boundary

A right medial parietal sensor showed a significant $\mathrm{COH}$ effect on the MEG amplitude between 350 and $400 \mathrm{~ms}$ after stimulus onset $\left(F_{(2,18)}=12.14, p<0.001\right)$ (Fig. 9A). This sensor also showed high amplitude in the difference topography (high-low $\mathrm{COH}$ ) (Fig. 9C). The time courses for the different $\mathrm{COH}$ levels in this time interval show higher amplitudes with increasing $\mathrm{COH}$ (Fig. 9D). Source localization during the epoch of interest localized the $\mathrm{COH}$ component in the right calcarine gyrus, assigned to BA17 with a probability of $90 \%$ [70-100\%], probability for BA18 was 30\% [20-50\%], central MNI coordinates $x, 8 ; y,-75 ; z, 7$. As for the sensor space, for the source space data, the right calcarine gyrus showed a significant $\mathrm{COH}$ effect on the amplitude $\left(F_{(2,18)}=\right.$ 11.70, $p<0.001$ ) (Fig. 9B). The differential (high-low $\mathrm{COH}$ ) sensor space MEG activity was not correlated with either the differential drift rate $(r=-0.045, p=0.90)$ (Fig. $9 E)$ or the differential boundary $(r=-0.54$, $p=0.11$ ) (Fig. 9F).

COH component 315 to $265 \mathrm{~ms}$ before the response in the left precentral gyrus correlated neither with the drift rate nor with the boundary

A left lateral frontal sensor showed a significant $\mathrm{COH}$ effect on the MEG amplitude between 315 and $265 \mathrm{~ms}$ before the response $\left(F_{(2,18)}=10.64, p<0.001\right)$ (Fig. 10A). This sensor also showed high activity in the difference topography (high-low $\mathrm{COH}$ ) (Fig. $10 C)$. The time courses for the different $\mathrm{COH}$ levels in this time interval show higher amplitudes under low $\mathrm{COH}$ than under medium or high $\mathrm{COH}$ (Fig. 10D). Source localization during the epoch of interest localized the $\mathrm{COH}$ component in the left precentral gyrus, BA6, central Talairach coordinates $x,-62 ; y, 2 ; z$, 21 (for labeling of prefrontal regions we used the Talairach Applet as the SPM8 Anatomy Toolbox provides maps in prefrontal regions for BA44 and 45 only; cf. Materials and Methods for further details). As for the sensor space, for the source space data, the left precentral gyrus showed a significant $\mathrm{COH}$ effect on the amplitude $\left(F_{(2,18)}=12.08, p<0.001\right)$ (Fig. $\left.10 B\right)$. The differential 
(high-low $\mathrm{COH}$ ) sensor space MEG activity was not highly correlated with either the differential drift rate $(r=-0.38, p=0.28)$ (Fig. 10E) or the differential boundary $(r=$ 0.37, $p=0.29)($ Fig. 10F).

\section{Discussion}

In the present study, we used MEG and a face-house categorization task to investigate how perceptual decision processes unfold in the human brain when emphasizing speed or accuracy of a decision (SAT) under different levels of sensory evidence $(\mathrm{COH})$. Emphasis on speed resulted in higher activation of SMA and precuneus whereas the left DLPFC showed the inverse pattern. When correlating these physiological effects with the boundary parameter, this dissociation was confirmed: changes in activity in right SMA were negatively correlated with the changes in response threshold (boundary), whereas changes in activity in left DLPFC were positively correlated with changes in boundary. Furthermore, we did not find SAT effects in sensory areas, i.e., bilateral fusiform gyrus and BA17 but these areas showed together with the right DLPFC enhanced activation for higher levels of sensory evidence. Activity in these areas was not correlated with the boundary.

\section{Behavioral and diffusion model data}

Behavioral data showed that subjects indeed traded speed for accuracy since they reacted faster at the cost of more errors when speed was emphasized. With decreasing sensory evidence the task became more difficult as RTs were slower and error rates higher. In the context of the diffusion model, congruent with a priori expectations, emphasis on accuracy compared with speed led to a significantly higher boundary while the drift rate was not significantly affected. The $\mathrm{COH}$ manipulation had a significant effect on the drift rate, but also on the boundary. The latter might seem surprising since, according to a classical interpretation of the diffusion model, the amount of sensory information would primarily affect the drift rate while changes in the speed-accuracy tradeoff would affect the boundary (Ratcliff and McKoon, 2008). However, several authors (Ditterich, 2006; Churchland et al., 2008; Cisek et al., 2009) have suggested variants of the sequential sampling model where the accumulator process does not only rely on sensory evidence but is complemented by an urgency signal that increases as a function of elapsed time and effectively reduces the boundary for decisions that take longer. Accordingly, subjects may have lowered their response threshold when sensory evidence was low resulting in a lower boundary estimate. In our data this effect seemed particularly pronounced in the accuracy condition but the interaction (between SAT and $\mathrm{COH}$ ) was far from significant $\left(F_{(2,18)}=1.80, p=0.19\right)$. This pattern, however, is congruent with results from Cisek et al. (2009). They showed that while initially the criterion of subjects to make a decision was higher when accuracy was emphasized compared with speed, the threshold
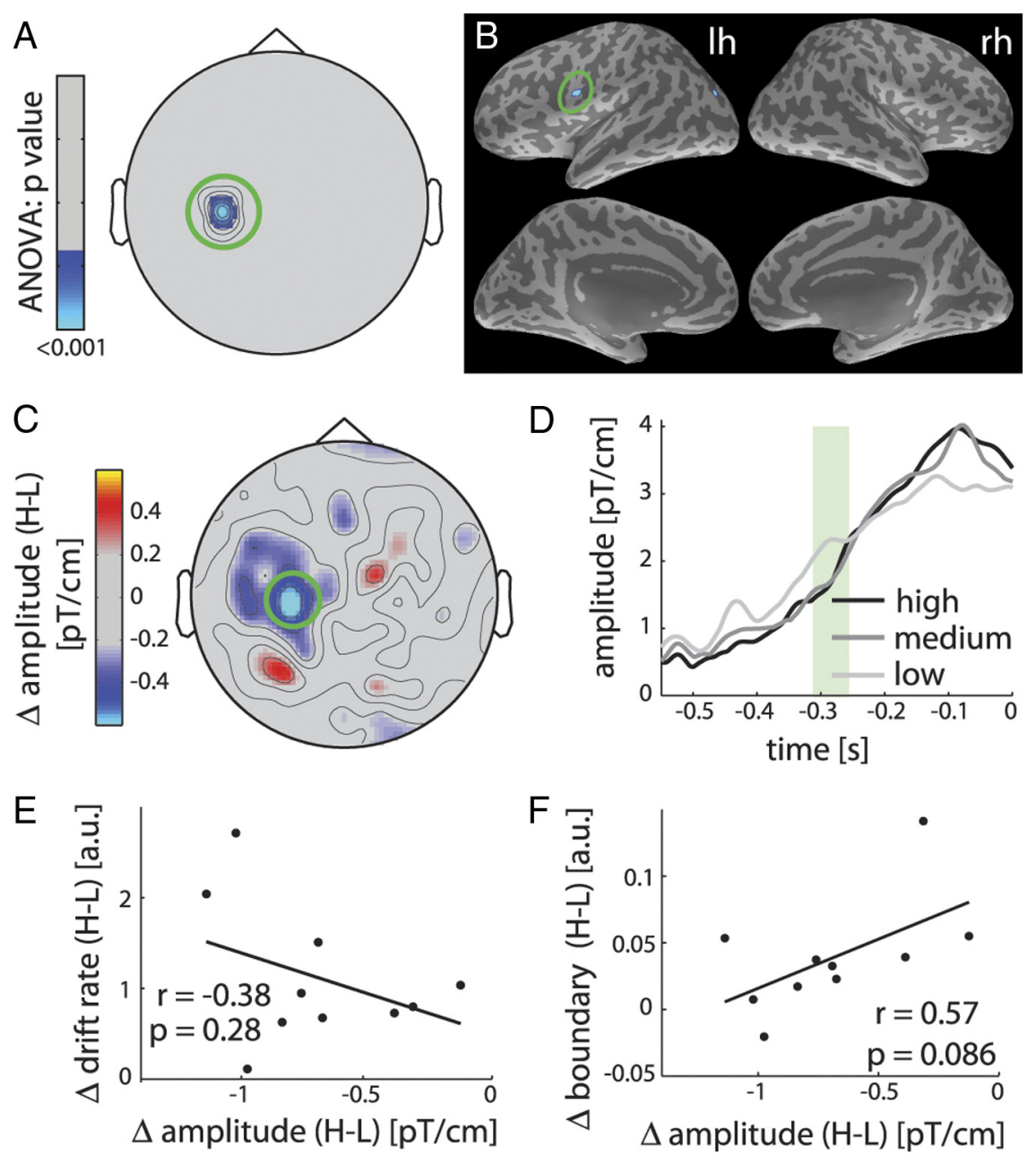

Figure 10. Significant $\mathrm{COH}$ effect between 315 and 265 ms before the response in left $\mathrm{BA} 6$ not correlated with the drift rate. $\boldsymbol{A}$, Significant $\mathrm{COH}$ effect on the MEG amplitude during the epoch if interest in a left laterofrontal sensor $\left(F_{(2,18)}=10.64, p<0.001\right)$. , Significant $\mathrm{COH}$ effect on the MEG amplitude in the source space $\left(F_{(2,18)}=12.08, p<0.001\right)$. The COH effect was located in the $\mathrm{COH}$. $\boldsymbol{E}$, No significant correlation between differential (high-low $\mathrm{COH}$ ) sensor space MEG activity and drift rate $(r=-0.38, p=$ 0.28). $\boldsymbol{F}$, Likewise, no significant correlation between differential MEG activity and boundary $(r=0.57, p=0.086)$.

was lowered at a steeper rate in the accuracy compared with the speed condition as time elapsed. This is convergent with the trend in our data. Future research should investigate the precise mechanisms behind such a dynamic threshold adaptation.

\section{Decision-related SAT component in the right SMA}

We measured higher activity levels under speed than accuracy between 275 and $325 \mathrm{~ms}$ after stimulus onset in the right SMA (Fig. 2A,B). The SMA seems to be involved in motor preparation as well as direct motor output (for review, see Nachev et al., 2008). Via connections with the basal ganglia, more precisely the striatum (Takada et al., 1998) or subthalamic nucleus (Nambu et al., 1996), it forms a circuit that is optimally suited to modulate action readiness, i.e., movement preparation or inhibition (Brinkman and Porter, 1979; Tanji and Kurata, 1982). Differential activations in pre-SMA and SMA were also found in SAT tasks using fMRI in humans. Forstmann et al. (2008) found greater activity under speed instructions in the right pre-SMA during the prestimulus period. van Veen et al. (2008) found greater activation before and less activation after stimulus onset 
in bilateral SMAs but overall activity was higher when emphasizing speed. Ivanoff et al. (2008) found higher stimulus-locked activation under speed instructions in right pre-SMA. In summary, greater activity under speed than accuracy instructions was found before or after stimulus onset.

Differential (accuracy-speed) activity in the right SMA was negatively correlated with the differential boundary, i.e., subjects with a low boundary difference showed higher difference in SMA activity (Fig. 2E). At first glance, this may seem at odds with an interpretation that the SMA is engaged in a process that sets the boundary. However, two hypotheses could explain this finding: (1) Enhanced SMA activity under speed conditions might be involved in overcoming the tonic inhibition provided by the output nuclei of the basal ganglia, thereby effectively lowering the boundary (Lo and Wang, 2006). (2) Enhanced SMA activity may be instrumental for a more efficient, i.e., faster, transformation of sensory signals into a motor response. In this context it would be of interest to study whether the connectivity of sensorimotor networks is enhanced under speed conditions.

\section{Decision-related SAT and COH components in the DLPFC}

Higher activity levels under accuracy than speed instructions were measured between 300 and $100 \mathrm{~ms}$ before the response in the left DLPFC, middle frontal gyrus (Fig. $5 A, B$ ). One may wonder if the amplitude differences between speed and accuracy 300 to $250 \mathrm{~ms}$ before the response are attributable to different reaction times (Fig. 5D). Between 150 and $100 \mathrm{~ms}$ before the response, however, the amplitudes under both instructions reached their maximum and the amplitude under accuracy was again significantly higher. This suggests that the observed differences reflect a combination of true differences in amplitude and relative onset latencies given the different mean RTs. Higher activation under accuracy than speed instructions in the middle frontal gyrus was also found by Forstmann et al. (2008). van Veen et al. (2008) reported greater activity under speed before stimulus onset in left DLPFC. Notably, their Figure 5 shows no higher peak activation after stimulus onset under the speed instruction.

The DLPFC receives input from secondary visual, auditory or somatosensory cortices and is connected with premotor structures including the SMA and the basal ganglia which makes it a candidate structure for the translation of higher-level sensory signals into motor commands (for review, see Miller and Cohen, 2001). Correspondingly, Kim and Shadlen (1999) in monkeys and Heekeren et al. (2004) in humans found the activity in left DLPFC to represent the differential output from neuronal populations representing the different stimulus alternatives. Hence, the DLPFC seems to be involved in computing the outcome of a decision or the subjective evidence for one alternative over the other. In that sense, the enhanced DLPFC activity for accuracy compared with speed conditions and its positive correlation with the boundary (Fig. 5E) might reflect a higher level of accumulated evidence.

While we did not find a significant effect of the $\mathrm{COH}$ manipulation on activity in the left DLPFC at a very conservative threshold, we found a significant one in the right DLPFC between 285 and $335 \mathrm{~ms}$ after stimulus onset (Fig. 8A,B). Differential activity in the right DLPFC in a task modulating sensory evidence was also found by Philiastides and Sajda (2007) using EEG-informed fMRI. Furthermore, differential (high-low $\mathrm{COH}$ ) activity in the right DLPFC correlated positively with the differential drift rate providing further evidence that the DLPFC integrates sensory information to compute a decision variable (Fig. $8 E$ ).

Summarizing, our results are consistent with the literature and add to the evidence that while activity in the left DLPFC was correlated with the boundary, activity in the right DLPFC was correlated with the drift rate.

\section{Other components characterizing SAT processing}

Higher activity levels under speed than accuracy were also measured in the left SMA, slightly later than in the right hemisphere and smaller in amplitude (Fig. $3 A, B, D$ ). A predominant right lateralization was expected since we included only correct answers after seeing pictures of faces in the analysis where subjects always responded with their left thumb. Likewise, intracranial EEG recordings (from epilepsy patients) preceding finger movements showed activity in bilateral SMAs with higher amplitudes in the contralateral hemisphere (Ikeda et al., 1992). Activity in the left SMA might be attributed to a general dominance of the left hemisphere in (pre-) motor processing (Kim et al., 1993). Differential activity in the left SMA did not correlate significantly with the differential boundary indicating that it was less directly involved in the decision process (Fig. $3 E$ ).

Furthermore, significantly higher activity levels under speed than accuracy were measured in the mesial extent of the precuneus, BA 31 (Fig. $4 A, B$ ). This region is, among others, functionally connected with the SMA via adjacent dorsal precuneus regions (Margulies et al., 2009), suggesting a role in (pre-) motor processing. In line with this, studies from Rushworth et al. (1998) and Bauer et al. (2009) suggest that the precuneus is involved in sensorimotor transformation processes. Like in the left SMA, differential activity in the precuneus did not correlate significantly with the differential boundary (Fig. 4E).

\section{Other components characterizing $\mathrm{COH}$ processing}

We measured activity that was parametrically modulated by $\mathrm{COH}$ in the following sensory regions: right and left fusiform gyrus $\sim 170 \mathrm{~ms}$ and in the right calcarine gyrus, BA17, $\sim 375 \mathrm{~ms}$ after stimulus onset (Figs. 6A, $B, 7 A, B, 9 A, B$ ). The M170 in the fusiform gyrus has repeatedly been shown to be related to processing of face stimuli (Swithenby et al., 1998; Liu et al., 2000). Late differential activity in BA17 might be due to activation of sensory feedback loops (Desmurget and Grafton, 2000; Lamme and Roelfsema, 2000). Importantly, none of the sensory areas showed SAT effects nor a correlation with diffusion model parameters. This does, however, not preclude modulations in neural activity due to SAT that the event-related field is not sensitive to, e.g., changes in induced rhythms or connectivity to premotor regions.

\section{Conclusion}

In conclusion, we show differential behavior of SMA and DLPFC activity under emphasis of speed against accuracy that can be interpreted such that the SMA under speed emphasis seems to facilitate fast responses by disinhibiting basal-ganglia-thalamic loops whereas the DLPFC may represent higher levels of accumulated evidence under emphasis of accuracy. Our results also show that the SMA seems dynamically active during stimulus processing, potentially reflecting an adaptive threshold regulation.

\section{References}

Bastiaansen MC, Knösche TR (2000) Tangential derivative mapping of axial MEG applied to event-related desynchronization research. Clin Neurophysiol 111:1300-1305.

Bauer M, Oostenveld R, Fries P (2009) Tactile stimulation accelerates behavioral responses to visual stimuli through enhancement of occipital gamma-band activity. Vision Res 49:931-942.

Bogacz R, Brown E, Moehlis J, Holmes P, Cohen JD (2006) The physics of optimal decision making: a formal analysis of models of performance in two-alternative forced-choice tasks. Psychol Rev 113:700-765. 
Bogacz R, Wagenmakers EJ, Forstmann BU, Nieuwenhuis S (2010) The neural basis of the speed-accuracy tradeoff. Trends Neurosci 33:10-16.

Brainard DH (1997) The Psychophysics Toolbox. Spat Vis 10:433-436.

Brinkman C, Porter R (1979) Supplementary motor area in the monkey: activity of neurons during performance of a learned motor task. J Neurophysiol 42:681-709.

Brodmann K (1909) Vergleichende Lokalisationslehre der Grosshirnrinde in ihren Prinzipien dargestellt auf Grund des Zellenbaues. Leipzig: Johann Ambrosius Barth Verlag.

Churchland AK, Kiani R, Shadlen MN (2008) Decision-making with multiple alternatives. Nat Neurosci 11:693-702.

Cisek P, Puskas GA, El-Murr S (2009) Decisions in changing conditions: the urgency-gating model. J Neurosci 29:11560-11571.

Dale AM, Fischl B, Sereno MI (1999) Cortical surface-based analysis. I. Segmentation and surface reconstruction. Neuroimage 9:179-194.

Desmurget M, Grafton S (2000) Forward modeling allows feedback control for fast reaching movements. Trends Cogn Sci 4:423-431.

Ditterich J (2006) Stochastic models of decisions about motion direction: behavior and physiology. Neural Netw 19:981-1012.

Fischl B, Sereno MI, Dale AM (1999a) Cortical surface-based analysis. II: Inflation, flattening, and a surface-based coordinate system. Neuroimage 9:195-207.

Fischl B, Sereno MI, Tootell RB, Dale AM (1999b) High-resolution intersubject averaging and a coordinate system for the cortical surface. Hum Brain Mapp 8:272-284.

Fischl B, Salat DH, Busa E, Albert M, Dieterich M, Haselgrove C, van der Kouwe A, Killiany R, Kennedy D, Klaveness S, Montillo A, Makris N, Rosen B, Dale AM (2002) Whole brain segmentation: automated labeling of neuroanatomical structures in the human brain. Neuron 33:341-355.

Forstmann BU, Dutilh G, Brown S, Neumann J, von Cramon DY, Ridderinkhof KR, Wagenmakers EJ (2008) Striatum and pre-SMA facilitate decisionmaking under time pressure. Proc Natl Acad Sci U S A 105:17538-17542.

Gold JI, Shadlen MN (2007) The neural basis of decision making. Annu Rev Neurosci 30:535-574.

Grosbras MH, Lobel E, Van de Moortele PF, LeBihan D, Berthoz A (1999) An anatomical landmark for the supplementary eye fields in human revealed with functional magnetic resonance imaging. Cereb Cortex 9:705-711.

Hämäläinen MS, Sarvas J (1989) Realistic conductivity geometry model of the human head for interpretation of neuromagnetic data. IEEE Trans Biomed Eng 36:165-171.

Haxby J, Hoffman E, Gobbini MI (2000) The distributed human neural system for face perception. Trends Cogn Sci 4:223-233.

Heekeren HR, Marrett S, Bandettini PA, Ungerleider LG (2004) A general mechanism for perceptual decision-making in the human brain. Nature 431:859-862.

Heekeren HR, Marrett S, Ruff DA, Bandettini PA, Ungerleider LG (2006) Involvement of human left dorsolateral prefrontal cortex in perceptual decision making is independent of response modality. Proc Natl Acad Sci U S A 103:10023-10028.

Heekeren HR, Marrett S, Ungerleider LG (2008) The neural systems that mediate human perceptual decision making. Nat Rev Neurosci 9:467-479.

Ikeda A, Lüders HO, Burgess RC, Shibasaki H (1992) Movement-related potentials recorded from supplementary motor area and primary motor area. Role of supplementary motor area in voluntary movements. Brain 115:1017-1043.

Ivanoff J, Branning P, Marois R (2008) fMRI evidence for a dual process account of the speed-accuracy tradeoff in decision-making. PloS One 3:e2635.

Johansen-Berg H, Behrens TE, Robson MD, Drobnjak I, Rushworth MF, Brady JM, Smith SM, Higham DJ, Matthews PM (2004) Changes in connectivity profiles define functionally distinct regions in human medial frontal cortex. Proc Natl Acad Sci U S A 101:13335-13340.

Kanwisher N, McDermott J, Chun MM (1997) The fusiform face area: a module in human extrastriate cortex specialized for face perception. J Neurosci 17:4302-4311.

Kim JN, Shadlen MN (1999) Neural correlates of a decision in the dorsolateral prefrontal cortex of the macaque. Nat Neurosci 2:176-185.

Kim SG, Ashe J, Hendrich K, Ellermann JM, Merkle H, Uğurbil K, Georgopoulos AP (1993) Functional magnetic resonance imaging of motor cortex: hemispheric asymmetry and handedness. Science 261:615-617.

Lamme VA, Roelfsema PR (2000) The distinct modes of vision offered by feedforward and recurrent processing. Trends Neurosci 23:571-579.

Liu J, Higuchi M, Marantz A, Kanwisher N (2000) The selectivity of the occipitotemporal M170 for faces. Neuroreport 11:337-341.
Lo CC, Wang XJ (2006) Cortico-basal ganglia circuit mechanism for a decision threshold in reaction time tasks. Nat Neurosci 9:956-963.

Margulies DS, Vincent JL, Kelly C, Lohmann G, Uddin LQ, Biswal BB, Villringer A, Castellanos FX, Milham MP, Petrides M (2009) Precuneus shares intrinsic functional architecture in humans and monkeys. Proc Natl Acad Sci U S A 106:20069-20074.

Miller EK, Cohen JD (2001) An integrative theory of prefrontal cortex function. Annu Rev Neurosci 24:167-202.

Nachev P, Kennard C, Husain M (2008) Functional role of the supplementary and pre-supplementary motor areas. Nat Rev Neurosci 9:856-869.

Nambu A, Takada M, Inase M, Tokuno H (1996) Dual somatotopical representations in the primate subthalamic nucleus: evidence for ordered but reversed body-map transformations from the primary motor cortex and the supplementary motor area. J Neurosci 16:2671-2683.

Osman A, Lou L, Muller-Gethmann H, Rinkenauer G, Mattes S, Ulrich R (2000) Mechanisms of speed-accuracy tradeoff: evidence from covert motor processes. Biol Psychol 51:173-199.

Pascual-Marqui RD (2002) Standardized low-resolution brain electromagnetic tomography (sLORETA): technical details. Methods Find Exp Clin Pharmacol 24 [Suppl D]:5-12.

Pascual-Marqui RD, Esslen M, Kochi K, Lehmann D (2002) Functional imaging with low-resolution brain electromagnetic tomography (LORETA): a review. Methods Find Exp Clin Pharmacol 24 [Suppl C]:91-95.

Philiastides MG, Sajda P (2007) EEG-informed fMRI reveals spatiotemporal characteristics of perceptual decision making. J Neurosci 27:13082-13091.

Philiastides MG, Ratcliff R, Sajda P (2006) Neural representation of task difficulty and decision making during perceptual categorization: a timing diagram. J Neurosci 26:8965-8975.

Ratcliff R (1978) A theory of memory retrieval. Psychol Rev 85:59-108.

Ratcliff R (2006) Modeling response signal and response time data. Cogn Psychol 53:195-237.

Ratcliff R, McKoon G (2008) The diffusion decision model: theory and data for two-choice decision tasks. Neural Comput 20:873-922.

Rinkenauer G, Osman A, Ulrich R, Muller-Gethmann H, Mattes S (2004) On the locus of speed-accuracy trade-off in reaction time: inferences from the lateralized readiness potential. J Exp Psychol Gen 133:261-282.

Rushworth MF, Johansen-Berg H, Young SA (1998) Parietal cortex and spatial-postural transformation during arm movements. J Neurophysiol 79:478-482.

Sangals J, Sommer W, Leuthold H (2002) Influences of presentation mode and time pressure on the utilisation of advance information in response preparation. Acta Psychol 109:1-24.

Ségonne F, Dale AM, Busa E, Glessner M, Salat D, Hahn HK, Fischl B (2004) A hybrid approach to the skull stripping problem in MRI. Neuroimage 22:1060-1075.

Shadlen MN, Newsome WT (2001) Neural basis of a perceptual decision in the parietal cortex (area LIP) of the rhesus monkey. J Neurophysiol 86:1916-1936.

Swithenby SJ, Bailey AJ, Bräutigam S, Josephs OE, Jousmäki V, Tesche CD (1998) Neural processing of human faces: a magnetoencephalographic study. Exp Brain Res 118:501-510.

Takada M, Tokuno H, Nambu A, Inase M (1998) Corticostriatal projections from the somatic motor areas of the frontal cortex in the macaque monkey: segregation versus overlap of input zones from the primary motor cortex, the supplementary motor area, and the premotor cortex. Exp Brain Res 120:114-128.

Talairach J, Tournoux P (1988) Co-planar stereotaxic atlas of the human brain: 3-dimensional proportional. Stuttgart: Thieme.

Tanji J, Kurata K (1982) Comparison of movement-related activity in two cortical motor areas of primates. J Neurophysiol 48:633-653.

Taulu S, Kajola M, Simola J (2004) Suppression of interference and artifacts by the Signal Space Separation Method. Brain Topogr 16:269-275.

Taulu S, Simola J, Kajola M (2005) Applications of the signal space separation method. IEEE Trans Signal Process 53:3359-3372.

van Veen V, Krug MK, Carter CS (2008) The neural and computational basis of controlled speed-accuracy tradeoff during task performance. J Cogn Neurosci 20:1952-1965.

Vandekerckhove J, Tuerlinckx F (2007) Fitting the Ratcliff diffusion model to experimental data. Psychon Bull Rev 14:1011-1026.

Vandekerckhove J, Tuerlinckx F (2008) Diffusion model analysis with MATLAB: a DMAT primer. Behav Res Methods 40:61-72. 